\title{
Restricted Structure Predictive Control For Linear and Nonlinear Systems
}

\author{
M.J. Grimble*/**, Pawel Majecki* \\ *Industrial Systems and Control Limited, 36 Renfield Street, Glasgow, G2 1LU, \\ Scotland, U.K., \\ (e-mail: pawel@isc-ltd.com) \\ **Industrial Control Centre, University of Strathclyde, 204 George Street, \\ Glasgow, G1 1XW, Scotland, U.K. \\ (e-mail: m.j.grimble@strath.ac.uk).
}

\begin{abstract}
An optimal predictive control algorithm is introduced for the control of linear and nonlinear discrete-time multivariable systems. The controller is specified in a "restricted structure" form involving a set of given linear transferfunctions and a set of gains that minimize a Generalized Predictive Control (GPC) cost-index. The set of functions can be chosen as proportional, integral and derivative terms; however, a wide range of controller structures is possible. This is referred to as Restricted-Structure GPC control.
\end{abstract}

The multi-step predictive control cost-function is novel, since it includes weightings on the "low-order" controller gains and the rate of change of gains. This considerably improves the numerical computations ensuring critical inverse computations cannot lead to a singular matrix. It also provides the option of adding soft or hard constraints on the controller gains which provides additional flexibility for control design. The ability to include a plant model that can include a general nonlinear operator is also new for restricted structure control solutions.

The low-order controller provides a potential improvement in robustness, since it is often less sensitive to plant uncertainties. The simple controller structure also enables relatively unskilled staff to retune the system using familiar tuning terms, and provides a potentially simpler $Q P$ problem for the constrained case.

Keywords: Nonlinear, restricted structure, predictive, minimum-variance, PID.

Acknowledgements: We are grateful for the cooperation between ISC Limited, Glasgow and the University of Strathclyde’s Industrial Control Centre. 


\section{Introduction}

The proposed Restricted Structure (RS) controller is an attempt to obtain the benefits of model based predictive control but using a low-order classical structure that can easily be retuned using familiar tuning terms. If adequate performance can be obtained the loworder controller often provides better robustness and can be implemented with lower computing resources. The PID controller is just one option for the choice of low-order controller structure, where the optimal gains are provided by the optimized solution.

The PID controller is very effective in industry and is used successfully across industrial sectors. However, if systems involve difficult dynamics, such as open-loop unstable or non-minimum phase behaviour, transport-delays, or interactions; then 'multiloop’ or 'decentralized’ PID control can provide poor performance. A higher-order controller may then be required and one that can deal formally with multivariable system dynamics. It is then reasonable to extend the controller structure by including other terms like a time-constant or a double integrator term.

Richalet developed simple approaches to Model Predictive Control (MPC), and introduced the idea of using a functional basis (Richalet et. al. 1978, Richalet et. al. 1993, Rossiter et. al. 2002, Richalet 1998). The first applications took place in the early 70's, and since that time there have been many applications Khadir and Ringwood (2008). The optimal control approach in the following also requires functions to be defined, but these relate to the structure of the controller to be implemented such as extended PID. The proposed approach is a special form of Generalized Predictive Control (GPC).

Optimal Restricted Structure (RS) feedback control design, involves a pre-defined controller structure, where gains are to be found to minimize a cost-index. The previous work by Grimble and co-workers (2000 and 2004 a, b, c) assumed a constant gain and 
controller parameters whereas the current work allows the gains to be time-varying. Moreover, the previous contributions assumed linear system models but in the following, a nonlinear model represented by an unstructured model is included.

The $R S$ multivariable controller is defined here in terms of a set of frequencysensitive functions, multiplied by gains that are found to minimise an extended GPC costfunction. The method will be referred to as Restricted Structure-Generalized Predictive Control (RS-GPC). In the RS-GPC approach introduced below the functions that determine the $R S$ controller are specified in the frequency-domain. The controller gains are computed to minimize a cost-index, which can penalize deviations in controller gain and the rate of change of these gains. The gains vary to compensate for any changes in the reference or disturbance signals.

There are many differences with traditional MPC theory, which may be listed as follows:

1. The restricted structure controller (within the control loop) is low-order, possibly half the usual order, which provides opportunities for improved robustness and a simplified on-line algorithm.

2. It may be used to tune classical controller structures (like auto-tuning).

3. The inclusion of gain and rate of change of gain terms in the criterion enables soft constraints to be applied to the controller parameter or gain-variations.

4. The feedback gains are optimized and not future control trajectories, so that the constrained version enables constraints on controller gains to be introduced, not available in standard MPC (the usual MPC constraints may also be included).

5. The nonlinear version described in the final sections allow a general nonlinear operator to represent the plant. 


\subsection{Methods of Computing Low-Order Optimal Controls}

A fixed-structure and low-order control scheme for Nano-positioning systems was developed by Eielsen et al. (2013). The authors noted "The control schemes are fixedstructure, low-order control laws, for which few results exist in the literature with regards to optimal tuning." There are powerful optimization algorithms or linear matrix inequality methods that have been applied to the problem. However, it is desirable that an optimal solution be physically justifiable and this requires a more direct solution.

Several attempts have been made to combine the benefits of PID with predictive control. In one approach the GPC performance index was modified by including PID terms (Guo et. al. 2008). A RS predictive control approach was described in (Grimble 2004c), but the numerical solution involved an approximation to a frequency response. A predictive PID controller was proposed in Katebi and Moradi (2001) and in Moradi, Katebi and Johnson (2001). The controller consisted of $m$ parallel PID controllers, where $m$ was the horizon chosen to give the best approximation to a GPC solution.

An optimal predictive PID control algorithm using a GPC solution was described in Udeuhi, Ordys and Grimble (2002). The aim was to develop an online optimization method for tuning PID controllers that could operate either as classical PID controllers or as a form of multivariable GPC controller. The controller involved weightings that were related to the PID controller gain terms after discretisation. The philosophy was to try to obtain the same performance from PID controls as with GPC design. This is not the aim below, but the $R S-G P C$ controller approach can be specialized to this case when the cost-function is simplified, and when the general functions that define the controller structure are based on PID control.

A GPC based PID controller for use in a weigh feeder, which dispenses material at a specified rate and is used in the process industries was proposed by Sato (2010). A stochastic predictive PID controller that was mathematically equivalent to a GPC 
controller with steady-state weighting was derived by Millar et. al. (1996), and a heat exchanger application was described.

The motivation in the following is to gain the benefits of low-order controllers, including the simplicity of implementation/tuning and the natural robustness they often inherit. The use of a predictive control framework to compute gains is a convenient optimization framework. It provides the benefits of model based control design. The plant model is novel for $R S$ control since it allows for the presence of an input subsystem represented by a general nonlinear operator. The cost-index used is also novel since it includes terms to limit the controller gain or parameter amplitudes, and to cost the rate of change of controller gains. It provides a unique ability to manage the optimal gains, using either soft or hard constraints, and it improves numerical properties.

\subsection{Strategy and Control Design Philosophy}

The discrete-time multivariable plant model is represented by the combination of a general linear or nonlinear operator and a linear state-space subsystem model (can be open-loop unstable). The process model includes a linear state-space model and any unstructured input subsystem which can include a nonlinear stable operator.

The objective here is not to generate a control action that is the same as GPC. The aim is to generate gains to minimise a GPC cost-index, under the constraint of using a $R S-G P C$ structure (like extended PID). A motivating factor is that classical controllers exhibit natural robustness properties in the presence of significant uncertainties and nonlinearities. The predictive element is concerned with providing a simple way of generating the $R S$-controller gains. An incidental benefit is that the controller will be able to exploit future set-point information and benefit from predictive capabilities. 


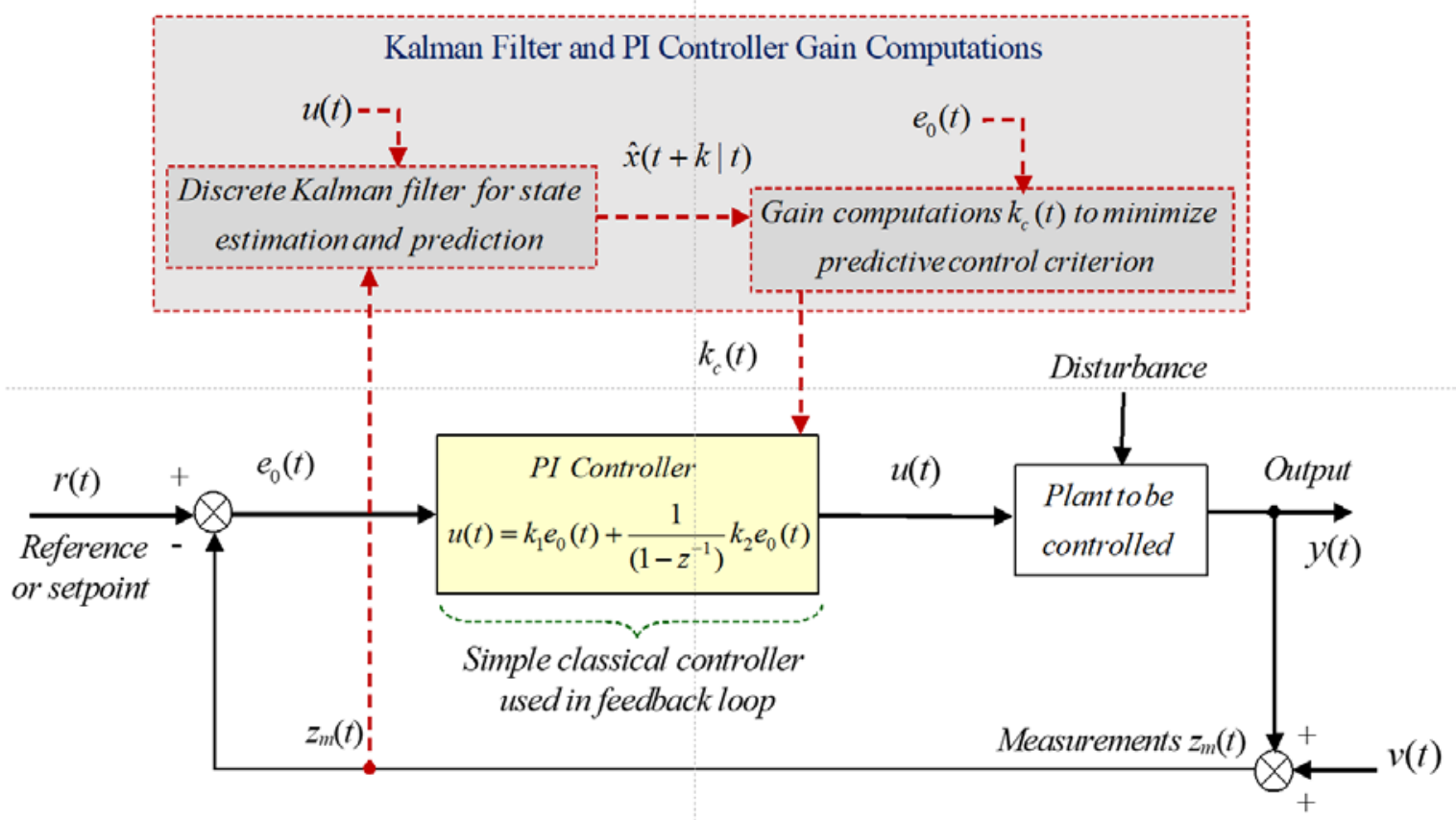

Figure 1. General Strategy of Restricted Structure Predictive Controller

The general philosophy is illustrated in Fig. 1. A low-order controller is chosen like the PI controller shown. A Kalman filter takes measurements and computed controls to determine the state estimates, which are used in an optimization algorithm to find the optimal PI controller gains stored in vector $k_{c}(t)$. The computation of the gains depends upon a receding horizon philosophy. This is different to the usual MPC algorithm since the controller within the loop has a conventional structure and $R S$-controller gains, rather than future controls, are computed.

\section{System Description}

The feedback system is shown in Fig. 2. The outputs to be controlled and measured outputs are denoted by $y(t)$ and $y_{m}(t)$, respectively. The observations includes measurement noise $z_{m}(t)=y_{m}(t)+v_{m}(t)$. The stochastic disturbance signals on measured and controlled outputs are represented by linear time-invariant models driven by zeromean white noise. The deterministic output disturbance terms and reference are denoted $d_{m}(t), d_{p}(t)$ and $r_{w}(t)$, respectively. These are known throughout the prediction-horizon. 
The white noise $v_{m}(t)$ is assumed to have a constant covariance matrix $R_{f}=R_{f}^{T} \geq 0$, and the zero-mean white noise disturbance $\xi(t)$ has an identity covariance matrix. The input sub-system $\mathcal{W}_{1}$ is assumed stable and has a general operator form, as follows:

$$
\left(\mathcal{W}_{1} u\right)(t)=z^{-k}\left(\mathcal{W}_{1 \mathrm{k}} u\right)(t)
$$

where $z^{-k} I$ denotes a matrix of the common delay elements in the output with $k>0$. Let $u_{0}(t)=\left(\mathcal{W}_{1 \mathrm{k}} u\right)(t)$ and denote the output linear subsystem as $W_{0}=z^{-k} W_{0 \mathrm{k}}$, which can contain any unstable modes. In the initial analysis, the strategy is to first consider the simpler linear problem (where $\mathcal{W}_{1 \mathrm{k}}=I$ ) and to then introduce the nonlinear inputsubsystem in the last section. The reference $r_{w}(t)$ is filtered so that $r(t)=W_{w}\left(z^{-1}\right) r_{w}(t)$, where $W_{w}\left(z^{-1}\right)$ is an ideal response model, and the error signal $e(t)=r(t)-y(t)$. The weighted error to be minimized in the cost-function is denoted:

$$
e_{p}(t)=P_{\mathrm{c}}\left(z^{-1}\right) e(t)
$$

where $P_{\mathrm{c}}\left(z^{-1}\right)$ is a stable proper dynamic cost-weighting. The input to the $R S$-controller is defined as follows:

$$
e_{0}(t)=r_{w}(t)-z_{m}(t)
$$

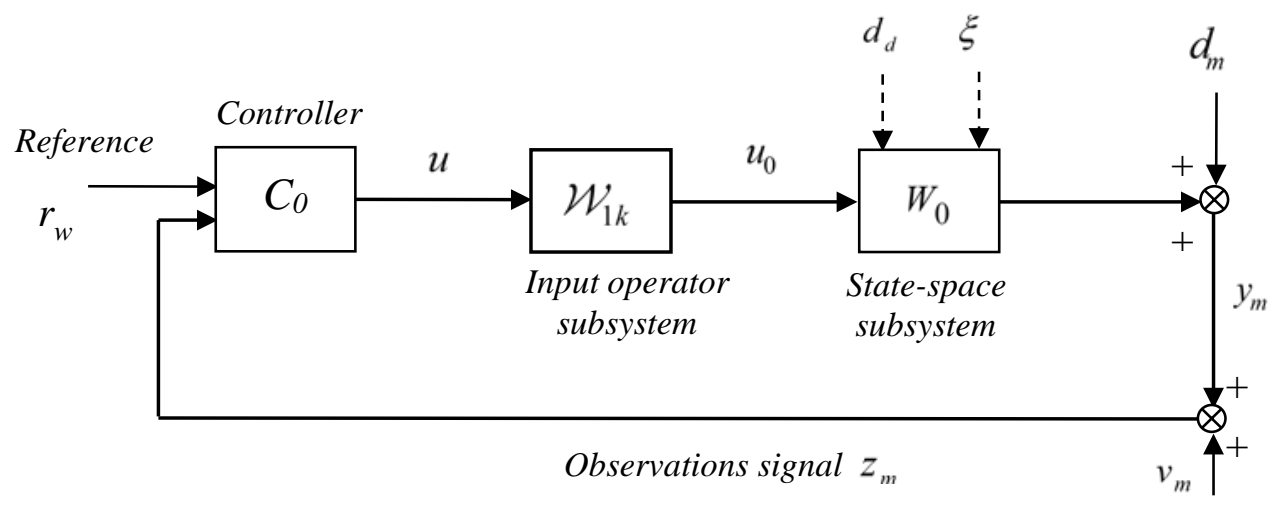


Figure 2. RS-GPC System for Unstructured and State-Space Plant Subsystems

\subsection{Augmented Linear State-Space Plant Output Subsystem}

The first subsystems to be defined is associated with the linear disturbance model and any linear state-space sub-system (denoted $W_{0}$ ) in the plant model. The state-space output subsystem is assumed stabilizable and detectable and is shown in Fig. 3. It includes any disturbance model and cost-function weighting term $P_{\mathrm{c}}\left(z^{-1}\right)$. The states, measured outputs, observations and weighted error of the augmented LTI system are given by the augmented system equations as follows:

$$
\begin{gathered}
x(t+1)=A x(t)+B u_{0}(t-k)+D \xi(t)+d_{d}(t) \\
y_{m}(t)=d_{m}(t)+C_{m} x(t)+E_{m} u_{0}(t-k) \\
z_{m}(t)=v_{m}(t)+d_{m}(t)+C_{m} x(t)+E_{m} u_{0}(t-k) \\
e_{p}(t)=d_{p}(t)+C_{p} x(t)+E_{p} u_{0}(t-k)
\end{gathered}
$$

The signals are explained in more detail and dimensions are listed in Appendix 1.

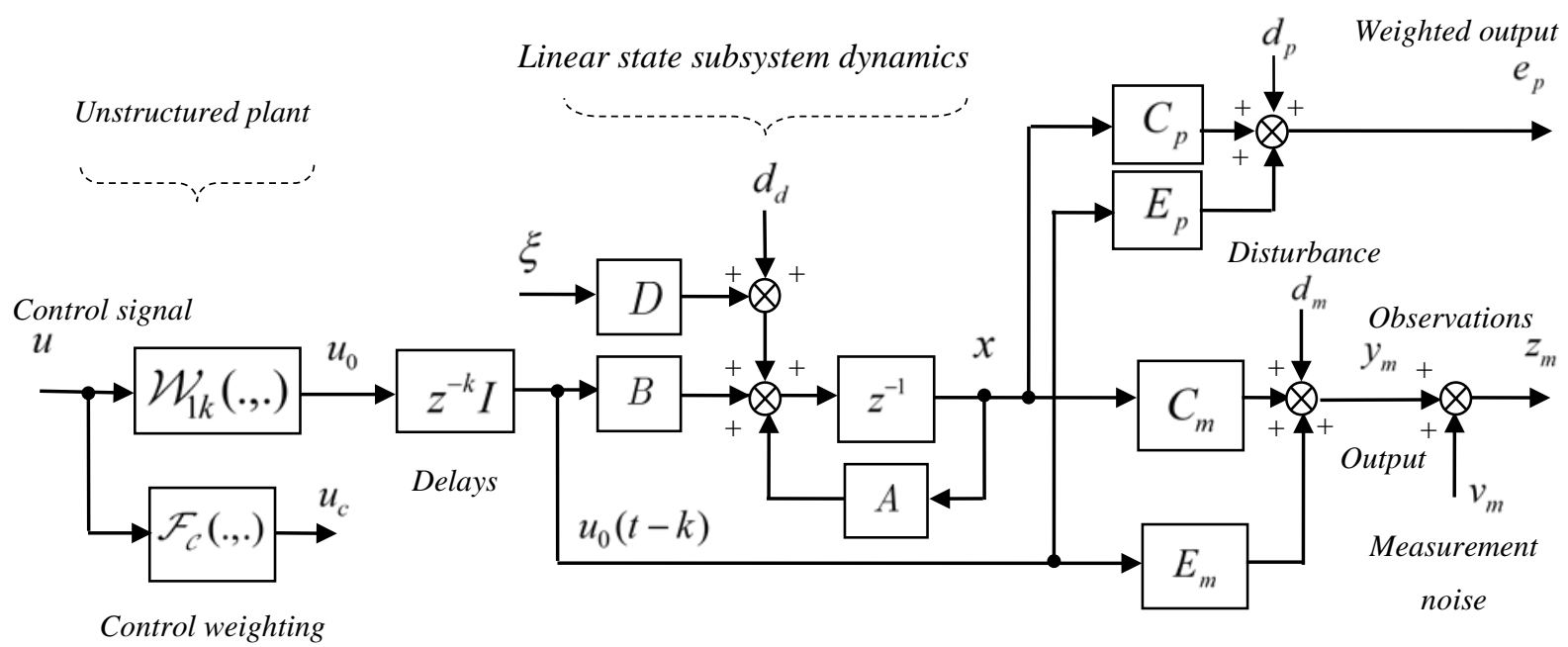

Fig. 3: Linear Plant Input and State-Space Linear Output and Disturbance Subsystems 


\subsection{State-Space Prediction Models}

The prediction of outputs is required in the later control solution. The future values of the states and outputs, at time $t$, may be obtained by repeated use of the state-equation:

$$
x(t+1)=A x(t)+B u_{0}(t-k)+D \xi(t)+d_{d}(t)
$$

Generalising this result, obtain the state at the future times $t+i$, where $i>0$, as:

$$
x(t+i)=A^{i} x(t)+\left(\sum_{j=1}^{i} A^{i-j}\left(B u_{0}(t+j-1-k)+D \xi(t+j-1)\right)\right)+d_{d d}(t+i-1)
$$

where the future known disturbance term is given as:

$$
d_{d d}(t+i-1)=\sum_{j=1}^{i} \mathcal{A}^{i-j} d_{d}(t+j-1)
$$

The future states depend upon the inputs and the state-vector at time $t$. The expression for the future states may be obtained by changing the time in (8) by the $k$-steps of the explicit delay giving:

$$
x(t+i+k)=A^{i} x(t+k)+\left(\sum_{j=1}^{i} A^{i-j}\left(B u_{0}(t+j-1)+D \xi(t+j+k-1)\right)\right)+d_{d d}(t+i+k-1)
$$

where $d_{d d}(t+i+k-1)=\sum_{j=1}^{i} \mathcal{A}^{i-j} d_{d}(t+j+k-1)$. The weighted error or output $e_{p}(t)$ to be regulated at future times can include any stable dynamic cost-function weighting. Noting (7) it has the following form (for $i \geq 1$ ):

$$
\begin{aligned}
& e_{p}(t+i+k)=d_{p}(t+i+k)+C_{p} x(t+i+k)+E_{p} u_{0}(t+i) \\
& =d_{p}(t+i+k)+C_{p} d_{d d}(t+i+k-1)+C_{p} A^{i} x(t+k)
\end{aligned}
$$




$$
+\sum_{j=1}^{i} C_{p} A^{i-j}\left(B u_{0}(t+j-1)+D \xi(t+j+k-1)\right)+E_{p} u_{0}(t+i)
$$

Collecting the deterministic disturbance signal terms together:

$$
d_{p d}(t+i+k)=d_{p}(t+i+k)+C_{p} d_{d d}(t+i+k-1)
$$

Weighted outputs or errors: Noting (11) the weighted output $e_{p}(t)$ becomes:

$$
\begin{gathered}
e_{p}(t+i+k)=d_{p d}(t+i+k)+C_{p} A^{i} x(t+k) \\
+\sum_{j=1}^{i} C_{p} A^{i-j}\left(B u_{0}(t+j-1)+D \xi(t+j+k-1)\right)+E_{p} u_{0}(t+i)
\end{gathered}
$$

State Prediction: The i-steps prediction may be written in terms of the future inputs and the estimated state-vector at time $t$ (using (8)) follows as:

$$
\hat{x}(t+i \mid t)=A^{i} \hat{x}(t \mid t)+\left(\sum_{j=1}^{i} A^{i-j} B u_{0}(t+j-1-k)\right)+d_{d d}(t+i-1)
$$

Vector Matrix Notation: Introducing an obvious notation for the error and output signals they may be collected in the $N+1$ vector form, where $N>0$ (Ordys and Clarke 1993) as:

$$
\begin{aligned}
& {\left[\begin{array}{c}
e_{p}(t+k) \\
e_{p}(t+1+k) \\
e_{p}(t+2+k) \\
\vdots \\
e_{p}(t+N+k)
\end{array}\right]=\left[\begin{array}{c}
d_{p d}(t+k) \\
d_{p d}(t+1+k) \\
d_{p d}(t+2+k) \\
\vdots \\
d_{p d}(t+N+k)
\end{array}\right]+\left[\begin{array}{c}
C_{p} I \\
C_{p} A \\
C_{p} A^{2} \\
\vdots \\
C_{p} A^{N}
\end{array}\right] x(t+k)} \\
& +\left[\begin{array}{cccc}
0 & 0 & \cdots & 0 \\
C_{p} B & 0 & \ddots & 0 \\
C_{p} A B & C_{p} B & & \\
\vdots & & \ddots & 0 \\
C_{p} A^{N-1} B & C_{p} A^{N-2} B & \cdots & C_{p} B
\end{array}\right]\left[\begin{array}{c}
u_{0}(t) \\
u_{0}(t+1) \\
\vdots \\
u_{0}(t+N-1)
\end{array}\right]
\end{aligned}
$$




$$
+\left[\begin{array}{c}
E_{p} u_{0}(t) \\
E_{p} u_{0}(t+1) \\
E_{p} u_{0}(t+2) \\
\vdots \\
E_{p} u_{0}(t+N)
\end{array}\right]+\left[\begin{array}{cccc}
0 & 0 & \cdots & 0 \\
C_{p} D & 0 & \ddots & 0 \\
C_{p} A D & C_{p} D & \ddots & \vdots \\
\vdots & \vdots & \ddots & 0 \\
C_{p} A^{N-1} D & C_{p} A^{N-2} D & \cdots & C_{p} D
\end{array}\right]\left[\begin{array}{c}
\xi(t+k) \\
\xi(t+1+k) \\
\vdots \\
\vdots \\
\xi(t+N-1+k)
\end{array}\right]
$$

This equation (15) may be written as follows:

$$
E_{P t+k, N}=D_{P t+k, N}+C_{P N} A_{N} x(t+k)+\left(C_{P N} B_{N}+E_{P N}\right) U_{t, N}^{0}+C_{P N} D_{N} W_{t+k, N}
$$

where the matrices are defined by comparison of (15) and (16). These are defined in Appendix 2. A matrix $V_{P N}$, for $N>0$, may also be defined as follows:

$$
V_{P N}=C_{P N} B_{N}+E_{P N}=\left[\begin{array}{ccccc}
E_{p} & 0 & \cdots & 0 & 0 \\
C_{p} B & E_{p} & \cdots & \vdots & 0 \\
\vdots & C_{p} B & \ddots & & \vdots \\
C_{p} A^{N-2} B & \vdots & \ddots & E_{p} & 0 \\
C_{p} A^{N-1} B & C_{p} A^{N-2} B & \cdots & C_{p} B & E_{p}
\end{array}\right]
$$

For a single-stage criterion the horizon $N=0$ and $V_{P N}=E_{p}$. The $k$ steps-ahead tracking error $E_{P t+k, N}$, includes any dynamic error weighting, and may be written, using (16), as:

$$
E_{P t+k, N}=D_{P t+k, N}+C_{P N} A_{N} x(t+k)+V_{P N} U_{t, N}^{0}+C_{P N} D_{N} W_{t+k, N}
$$

\subsection{Linear Prediction Equations}

The $i$-steps ahead prediction of the output may be computed noting (11) and assuming the future values of the control action are known. Let $\hat{e}_{p}(t+i+k \mid t)=E\left\{\hat{e}_{p}(t+i+k) \mid t\right\}$, then the predicted weighted signal to be minimized, using (13), becomes:

$$
\hat{e}_{p}(t+i+k \mid t)=d_{p d}(t+i+k)+C_{p} A^{i} \hat{x}(t+k \mid t)+\sum_{j=1}^{i} C_{p} A^{i-j} B u_{0}(t+j-1)+E_{p} u_{0}(t+i)
$$


The $\hat{x}(t+k \mid t)$ denotes a least squares state estimate from a Kalman filter, driven by measured outputs (6). Collecting results for the case $N \geq 0$ the vector of predicted outputs $\hat{E}_{P t+k, N}$ may be obtained in the block matrix form:

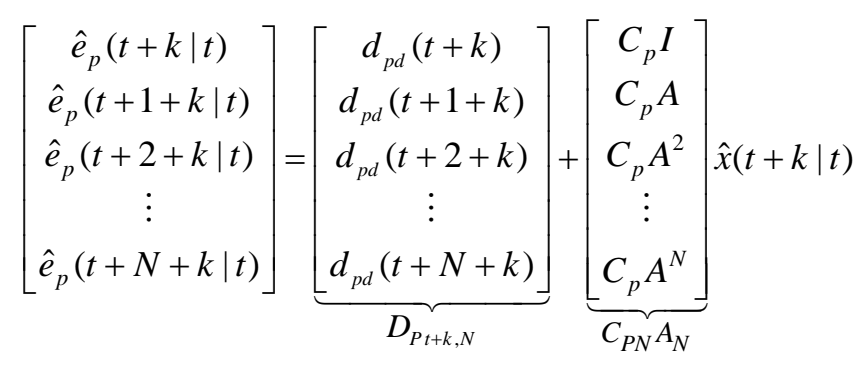

$$
\begin{aligned}
& +\underbrace{\left[\begin{array}{ccccc}
E_{p} & 0 & \cdots & 0 & 0 \\
C_{p} B & E_{p} & \cdots & \vdots & 0 \\
\vdots & C_{p} B & \ddots & & \vdots \\
C_{p} A^{N-2} B & \vdots & \ddots & E_{p} & 0 \\
C_{p} A^{N-1} B & C_{p} A^{N-2} B & \cdots & C_{p} B & E_{p}
\end{array}\right]}_{V_{P N}=C_{P N} B_{N}+E_{P N}} \underbrace{\left[\begin{array}{c}
u_{0}(t) \\
u_{0}(t+1) \\
\vdots \\
u_{0}(t+N)
\end{array}\right]}_{U_{t, N}^{0}}
\end{aligned}
$$

This prediction $N+1$ vector in (20) can clearly be written in the form:

$$
\hat{E}_{P t+k, N}=D_{P t+k, N}+C_{P N} A_{N} \hat{x}(t+k \mid t)+V_{P N} U_{t, N}^{0}
$$

\section{Output prediction error:}

$$
\begin{gathered}
\tilde{E}_{P t+k, N}=E_{P t+k, N}-\hat{E}_{P t+k, N} \\
=C_{P N} A_{N} x(t+k)+V_{P N} U_{t, N}^{0}+C_{P N} D_{N} W_{t+k, N}-\left(C_{P N} A_{N} \hat{x}(t+k \mid t)+V_{P N} U_{t, N}^{0}\right)
\end{gathered}
$$

Thence, the inferred output estimation error has the form:

$$
\tilde{E}_{P t+k, N}=C_{P N} A_{N} \tilde{x}(t+k \mid t)+C_{P N} D_{N} W_{t+k, N}
$$

where the $k$-steps-ahead state estimation error $\tilde{x}(t+k \mid t)=x(t+k)-\hat{x}(t+k \mid t) \quad$ is independent of the choice of control. Also recall $\hat{x}(t+k \mid t)$ and $\tilde{x}(t+k \mid t)$ are orthogonal 
and the expectation of the product of the future values of the control action (assumed known in deriving the prediction equation), and the zero mean white noise driving signals, is null. It follows that $\hat{E}_{P t+k, N}$ in (21) and the prediction error $\tilde{E}_{P t+k, N}$ are orthogonal.

\subsection{Kalman Estimator}

The state estimate $\hat{x}(t+k \mid t)$ may be obtained, $k$-steps-ahead, in a computationally efficient form from a Kalman filter (Grimble and Johnson, 1988). The number of states in the filter is not increased by the number of the explicit delays $k$. The estimation equations may be listed as follows:

$$
\begin{gathered}
\hat{x}(t+1 \mid t)=A \hat{x}(t \mid t)+B u_{0}(t-k)+d_{d}(t) \\
\hat{x}(t+1 \mid t+1)=\hat{x}(t+1 \mid t)+K_{f}\left(z_{m}(t+1)-\hat{z}_{m}(t+1 \mid t)\right) \\
\hat{z}_{m}(t+1 \mid t)=d_{m}(t+1)+C_{m} \hat{x}(t+1 \mid t)+E_{m} u_{0}(t+1-k)
\end{gathered}
$$

where

\section{Restricted Structure-Generalized Predictive Control}

To parameterize the controller a total of $N_{e}$ linear dynamic functions can be chosen with different frequency responses. It may be useful to introduce pre and post-compensation matrices, $L_{u}\left(z^{-1}\right)$ and $L_{e}\left(z^{-1}\right)$, so that the control signal may be expressed as follows:

$$
u(t)=L_{u}\left(z^{-1}\right) \sum_{j=1}^{N_{e}} f_{j}\left(z^{-1}, k_{j}(t)\right) L_{e}\left(z^{-1}\right) e_{0}(t)=L_{u}\left(z^{-1}\right) \sum_{j=1}^{N_{e}} f_{j}\left(z^{-1}, k_{j}(t)\right) e_{L}(t)
$$

where the weighted input to the RS-controller:

$$
e_{L}(t)=L_{e}\left(z^{-1}\right) e_{0}(t)=L_{e}\left(z^{-1}\right)\left(r_{w}(t)-z(t)\right)
$$

The $L_{u}\left(z^{-1}\right)$ and $L_{e}\left(z^{-1}\right)$ denotes frequency weighting on plant input and outputs. These 
weightings are not essential but they may be useful for multivariable systems when diagonal functions are used to simplify (26). The details of the controller parameterization in (26), and the matrices involved, are described in Appendix 3. It is shown that the gains of the controller in the restricted structure controller can be collected in a vector denoted $k_{c}(t)$. The $R S$-controller may then be written in the following form:

$$
u(t)=L_{u}\left(z^{-1}\right) F_{e}(t) k_{c}
$$

The gains might for example, represent the vector of gains in a 3-term PID controller.

\subsection{Restricted Structure Controller}

There are two methods of implementing the restricted structure controller. The gain can be written in terms of a fixed gain and a deviation. That is, for the optimization procedure the gains can be separated into a constant component $\bar{k}_{c}$ and a time-varying deviation $\tilde{k}_{c}(t)$, where the total gain:

$$
k_{c}(t)=\bar{k}_{c}+\tilde{k}_{c}(t)
$$

This gives rise to two cases:

1. Letting $\bar{k}_{c}=0$ is what will be termed the absolute control gain case, where the total controller gains $k_{c}(t)=\tilde{k}_{c}(t)$ are to be computed to minimize the criterion.

2. If $\bar{k}_{c} \neq 0$ the so-called gain, deviation $\tilde{k}_{c}(t)$ is to be computed to minimize the criterion.

If a PID controller structure is chosen, then the first case above is where the total controller gains are to be minimized. The second case can be used when an existing PID controller is involved, which defines the fixed gain $\bar{k}_{c}$ term. The computed gain is then just the deviation away from the fixed PID levels. This case can be thought of as using 
two parallel PID controllers, with one having fixed gains, and one having gain deviations. The RS controller may be written, using (26) and (29) as follows:

$$
u(t)=L_{u}\left(z^{-1}\right) \sum_{j=1}^{N_{e}}\left\{f_{j}\left(z^{-1}\right) \bar{k}_{j} e_{L}(t)\right\}+L_{u}\left(z^{-1}\right) \sum_{j=1}^{N_{e}}\left\{f_{j}\left(z^{-1}\right) \tilde{k}_{j} e_{L}(t)\right\}
$$

In terms of the parametrization and the matrix $F_{e}(t)$ introduced in Appendix 3, the $R S$-control follows as:

$$
u(t)=L_{u}\left(z^{-1}\right) F_{e}(t) k_{c}=L_{u}\left(z^{-1}\right) F_{e}(t) \bar{k}_{c}+L_{u}\left(z^{-1}\right) F_{e}(t) \tilde{k}_{c}(t)
$$

\subsection{Vector of Future Controls}

The computation of the controller gains in the next section, based on a predictive control philosophy, provides the gains in a simple manner. This is not the usual approach to predictive control, since it will be assumed that the controller structure is defined in a desired form a priori. A modified receding-horizon philosophy will be invoked. Recall an optimal control signal at time $t$ is based on the receding horizon principle (Kwon and Pearson, 1977), where the optimal control is taken as the first element in vector $U_{t, N}^{0}$. The optimal control is computed for the full horizon but only the value at time $t$ is used.

The equivalent assumption for RS-GPC control is that $k_{c}(t)$ can be assumed constant in the interval $[0, N]$ and the computed $k_{c}(t)$ can be used to compute the optimal control for time $t$. In the spirit of receding control at the next sample time the process can be repeated and a new gain can be computed and used to compute the optimal control. With this assumption the vector of future controls $U_{t, N}$ may be written, using (83), as follows:

$$
U_{t, N}=\left[\begin{array}{c}
u(t) \\
u(t+1) \\
\vdots \\
u(t+N)
\end{array}\right]=\left[\begin{array}{c}
L_{u}\left(z^{-1}\right) F_{e}(t) \\
L_{u}\left(z^{-1}\right) F_{e}(t+1) \\
\vdots \\
L_{u}\left(z^{-1}\right) F_{e}(t+N)
\end{array}\right] k_{c}(t)
$$


At each future time the gain in (31) is assumed the same over the prediction horizon. This is different to conventional $M P C$, where the vector of future controls is computed. The matrix (31) may be denoted $U_{f e}$ and defined as follows:

$$
U_{f e}(t)=\left[\begin{array}{llll}
\left(L_{u}\left(z^{-1}\right) F_{e}(t)\right)^{T} & \left(L_{u}\left(z^{-1}\right) F_{e}(t+1)\right)^{T} \quad \cdots \quad\left(L_{u}\left(z^{-1}\right) F_{e}(t+N)\right)^{T}
\end{array}\right]^{T}
$$

The vector of future controls for the RS-GPC controller, from (31) and (32):

$$
U_{t, N}=U_{f e}(t) k_{c}(t)
$$

\section{Optimizing the Restricted-Structure Controller}

The minimization of a cost-function for a controller of restricted structure, is well established, but the $R S$ problem considered below is unusual. First, there is no approximation in the optimization procedure that occurs in (Grimble, 2004a, 2004b). Secondly, the controller structure is defined in a form where functions are pre-specified and are multiplied by gains that are to be optimized. For the initial results, the unstructured subsystem block is removed by letting $\mathcal{W}_{1 \mathrm{k}}=I$. It is reintroduced in Section §6. The GPC performance index that motivates the RS-GPC criterion described below, may be expressed as follows (see Clarke et. al. 1987, 1989):

$$
\left.J=E\left\{\sum_{j=0}^{N} \mathrm{e}_{p}(t+j+k)^{T} \mathrm{e}_{p}(t+j+k)+\lambda_{j}^{2} u_{0}(t+j)^{T} u_{0}(t+j)\right) \mid t\right\}
$$

where $E\{. \mid t\}$ denotes the conditional expectation, conditioned on measurements up to time $t$ and $\lambda_{j}$ denotes a scalar control signal weighting. The optimal control signal is to be calculated for the interval $\tau \in[t, t+N]$. The state-space model generating the tracking error $e_{p}$ may include any dynamic cost-function weighting $P_{c}\left(z^{-1}\right)$, such as a low-pass 
filter to penalise the low-frequency disturbances. The GPC criterion may be written using the previous definitions of future signals as follows:

$$
J=E\left\{J_{t} \mid t\right\}=E\left\{E_{P t+k, N}^{T} E_{P t+k, N}+U_{t, N}^{0 T} \Lambda_{N}^{2} U_{t, N}^{0} \mid t\right\}
$$

The RS-GPC cost-function required here has a term to limit the deviation in gains of the controller that may be added into (35), so that large gain deviations are penalized. In addition to be able to be able to influence the rate of gain variations the difference of the gain deviations may also be costed. The RS-GPC cost-function is defined as follows:

$$
J=E\left\{J_{t} \mid t\right\}=E\left\{E_{P t+k, N}^{T} E_{P t+k, N}+U_{t, N}^{0 T} \Lambda_{N}^{2} U_{t, N}^{0}+\tilde{k}_{c}^{T}(t) \Lambda_{K}^{2} \tilde{k}_{c}(t)+\Delta \tilde{k}_{c}^{T}(t) \Lambda_{D}^{2} \Delta \tilde{k}_{c}(t) \mid t\right\}
$$

where the gain change deviation:

$$
\Delta \tilde{k}_{c}(t)=\tilde{k}_{c}(t)-\tilde{k}_{c}(t-1)=k_{c}(t)-k_{c}(t-1)
$$

The terms in the criterion may be summarized as follows:

- The cost-weightings on the future inputs $u_{0}$ are defined as:

$$
\Lambda_{N}^{2}=\operatorname{diag}\left\{\lambda_{0}^{2}, \lambda_{1}^{2}, \ldots, \lambda_{N}^{2}\right\}
$$

- The cost-weightings on the deviations in controller gains are defined as:

$$
\Lambda_{K}^{2}=\operatorname{diag}\left\{\rho_{0}^{2}, \rho_{1}^{2}, \ldots, \rho_{N_{e}}^{2}\right\} .
$$

- The cost-weighting on the deviations in the difference of the gains is denoted:

$$
\Lambda_{D}^{2}=\operatorname{diag}\left\{\gamma_{0}^{2}, \gamma_{1}^{2}, \ldots, \gamma_{N_{e}}^{2}\right\}
$$

where the integer $N$ is the number of steps in the prediction horizon and $N_{e}$ is the number of functions employed in parameterizing the controller. 
Implementing the controller gains in the parallel form in (29) can be interpreted as the first term being a fixed controller and the second term (having optimal deviation gains) as providing adaption to reference or disturbance signal changes. The cost-function (36) includes a penalty on the gain deviations $\tilde{k}_{c}(t)$ and their rate of change $\Delta \tilde{k}_{c}(t)$. The two methods of implementing the controller gains will not therefore lead to the same results. For example, assuming the fixed component of the controller is stabilizing and increasing the penalty on $\tilde{k}_{c}(t)$ will result in the fixed controller performance being approached.

\subsection{Cost-Function Minimization}

The vector of future errors can be replaced by orthogonal predicted errors and estimation error terms. From equation (36) obtain the criterion as follows:

$$
\begin{gathered}
J=E\left\{\left(\hat{E}_{P t+k, N}+\tilde{E}_{P t+k, N}\right)^{T}\left(\hat{E}_{P t+k, N}+\tilde{E}_{P t+k, N}\right)+U_{t, N}^{0 T} \Lambda_{N}^{2} U_{t, N}^{0}\right. \\
\left.+\tilde{k}_{c}^{T}(t) \Lambda_{K}^{2} \tilde{k}_{c}(t)+\Delta \tilde{k}_{c}^{T}(t) \Lambda_{D}^{2} \Delta \tilde{k}_{c}(t) \mid t\right\}
\end{gathered}
$$

The terms in the cost-index can be simplified by using the orthogonality of the optimal estimate $\hat{E}_{P t+k, N}$ and the estimation error $\tilde{E}_{P t+k, N}$. Simplifying the expression,

$$
J=\hat{E}_{P t+k, N}^{T} \hat{E}_{P t+k, N}+U_{t, N}^{0 T} \Lambda_{N}^{2} U_{t, N}^{0}+\tilde{k}_{c}^{T}(t) \Lambda_{K}^{2} \tilde{k}_{c}(t)+\Delta \tilde{k}_{c}^{T}(t) \Lambda_{D}^{2} \Delta \tilde{k}_{c}(t)+J_{0}(t)
$$

where $\tilde{E}_{P t+k, N}=C_{P N} A_{N} \tilde{x}(t+k \mid t)+C_{P N} D_{N} W_{t+k, N}$ and the cost-term $J_{0}(t)=E\left\{\tilde{E}_{P t+k, N}^{T} \tilde{E}_{P t+k, N} \mid t\right\}$ is independent of the control. Noting (21) the vector of state-estimates may be written as follows:

$$
\hat{E}_{P t+k, N}=D_{P t+k, N}+C_{P N} A_{N} \hat{x}(t+k \mid t)+V_{P N} U_{t, N}^{0}=\tilde{D}_{P t+k, N}+V_{P N} U_{t, N}^{0}
$$

where the signal: 


$$
\tilde{D}_{P t+k, N}=D_{P t+k, N}+C_{P N} A_{N} \hat{x}(t+k \mid t)
$$

The state-estimate $\hat{x}(t+k \mid t)$ only depends upon past values of the control signal. The multi-step cost-function (39) may therefore be expanded as follows:

$$
\begin{gathered}
J=\left(\tilde{D}_{P t+k, N}+V_{P N} U_{t, N}^{0}\right)^{T}\left(\tilde{D}_{P t+k, N}+V_{P N} U_{t, N}^{0}\right)+U_{t, N}^{0 T} \Lambda_{N}^{2} U_{t, N}^{0} \\
+\tilde{k}_{c}^{T}(t) \Lambda_{K}^{2} \tilde{k}_{c}(t)+\Delta \tilde{k}_{c}^{T}(t) \Lambda_{D}^{2} \Delta \tilde{k}_{c}(t)+J_{0}(t) \\
=\tilde{D}_{P t+k, N}^{T} \tilde{D}_{P t+k, N}+U_{t, N}^{0 T} V_{P N}^{T} \tilde{D}_{P t+k, N}+\tilde{D}_{P t+k, N}^{T} V_{P N} U_{t, N}^{0}+U_{t, N}^{0 T}\left(V_{P N}^{T} V_{P N}+\Lambda_{N}^{2}\right) U_{t, N}^{0} \\
+\tilde{k}_{c}^{T}(t) \Lambda_{K}^{2} \tilde{k}_{c}(t)+\Delta \tilde{k}_{c}^{T}(t) \Lambda_{D}^{2} \Delta \tilde{k}_{c}(t)+J_{0}(t)
\end{gathered}
$$

Before performing the optimization, the controller structure will be defined to have the desired restricted structure form. From (29) $k_{c}(t)=\bar{k}_{c}+\tilde{k}_{c}(t)$ and from (37) the change in gain $\Delta \tilde{k}_{c}(t)=k_{c}(t)-k_{c}(t-1)$. Recall in this section is $\mathcal{W}_{1 \mathrm{k}}=I$, so that $U_{t, N}^{0}=U_{t, N}$, where $U_{t, N}=U_{f e}(t) k_{c}(t)$. Substituting the cost-function (42) may now be expanded as below:

$$
\begin{aligned}
J=\tilde{D}_{P t+k, N}^{T} \tilde{D}_{P t+k, N}+k_{c}^{T}(t) U_{f e}^{T} V_{P N}^{T} \tilde{D}_{P t+k, N}+\tilde{D}_{P t+k, N}^{T} V_{P N} U_{f e} k_{c}(t)+k_{c}^{T}(t)\left(U_{f e}^{T}\left(V_{P N}^{T} V_{P N}+\Lambda_{N}^{2}\right) U_{f e}\right) k_{c}(t) \\
-\bar{k}_{c}^{T} \Lambda_{K}^{2} k_{c}(t)-k_{c}^{T}(t) \Lambda_{K}^{2} \bar{k}_{c}-k_{c}^{T}(t-1) \Lambda_{D}^{2} k_{c}(t)-k_{c}^{T}(t) \Lambda_{D}^{2} k_{c}(t-1) \\
+k_{c}^{T}(t)\left(\Lambda_{K}^{2}+\Lambda_{D}^{2}\right) k_{c}(t)+\bar{k}_{c}^{T} \Lambda_{K}^{2} \bar{k}_{c}+k_{c}^{T}(t-1) \Lambda_{D}^{2} k_{c}(t-1)+J_{0}
\end{aligned}
$$

The equations can be simplified by defining:

$$
\begin{gathered}
X_{N}=U_{f e}^{T}\left(V_{P N}^{T} V_{P N}+\Lambda_{N}^{2}\right) U_{f e}+\Lambda_{K}^{2}+\Lambda_{D}^{2} \\
P_{C N}=U_{f e}^{T} V_{P N}^{T}
\end{gathered}
$$




$$
C_{\phi}=P_{C N} C_{P N} A_{N}=U_{f e}^{T} V_{P N}^{T} C_{P N} A_{N}
$$

Substituting for these system matrices, the following expression is obtained:

$$
\begin{gathered}
J=\tilde{D}_{P t+k, N}^{T} \tilde{D}_{P t+k, N}+k_{c}^{T}(t) P_{C N} \tilde{D}_{P t+k, N}+\tilde{D}_{P t+k, N}^{T} P_{C N}^{T} k_{c}(t) \\
-\bar{k}_{c}^{T} \Lambda_{K}^{2} k_{c}(t)-k_{c}^{T}(t) \Lambda_{K}^{2} \bar{k}_{c}-k_{c}^{T}(t-1) \Lambda_{D}^{2} k_{c}(t)-k_{c}^{T}(t) \Lambda_{D}^{2} k_{c}(t-1) \\
+k_{c}^{T}(t)\left(U_{f e}^{T}\left(V_{P N}^{T} V_{P N}+\Lambda_{N}^{2}\right) U_{f e}+\Lambda_{K}^{2}+\Lambda_{D}^{2}\right) k_{c}(t)+\bar{k}_{c}^{T} \Lambda_{K}^{2} \bar{k}_{c}+k_{c}^{T}(t-1) \Lambda_{D}^{2} k_{c}(t-1)+J_{0} \\
=\tilde{D}_{P t+k, N}^{T} \tilde{D}_{P t+k, N}+k_{c}^{T}(t) P_{C N} \tilde{D}_{P t+k, N}+\tilde{D}_{P t+k, N}^{T} P_{C N}^{T} k_{c}(t) \\
-\left(\bar{k}_{c}^{T} \Lambda_{K}^{2}+k_{c}^{T}(t-1) \Lambda_{D}^{2}\right) k_{c}(t)-k_{c}^{T}(t)\left(\Lambda_{K}^{2} \bar{k}_{c}+\Lambda_{D}^{2} k_{c}(t-1)\right)+k_{c}^{T}(t) X_{N} k_{c}(t)+\bar{J}_{0}
\end{gathered}
$$

Let the signal $\psi(t)$ be defined to simplify this equation:

$$
\psi(t)=-\Lambda_{K}^{2} \bar{k}_{c}-\Lambda_{D}^{2} k_{c}(t-1)
$$

The cost-function expression becomes:

$$
\begin{gathered}
J=\tilde{D}_{P t+k, N}^{T} \tilde{D}_{P t+k, N}+k_{c}^{T}(t) P_{C N} \tilde{D}_{P t+k, N}+\tilde{D}_{P t+k, N}^{T} P_{c N}^{T} k_{c}(t) \\
+\psi(t)^{T} k_{c}(t)+k_{c}^{T}(t) \psi(t)+k_{c}^{T}(t) X_{N} k_{c}(t)+\bar{J}_{0} \\
\bar{J}_{0}=\bar{k}_{c}^{T} \Lambda_{K}^{2} \bar{k}_{c}+k_{c}^{T}(t-1) \Lambda_{D}^{2} k_{c}(t-1)+J_{0}
\end{gathered}
$$

and $J_{0}$ (defined in (39)) are both terms that determine the minimum cost (independent of the gains $k_{c}(t)$ to be optimized). The approach for minimizing this cost term, if the signals are deterministic, is almost identical to that when the conditional cost-function is considered. The gradient of the cost-function must be set to zero, to obtain the vector of 
future optimal controls (Grimble and Johnson, 1988). Noting the $\bar{J}_{0}$ term is independent of the control action, the vector of optimal gains becomes:

$$
k_{c}(t)=-\left(U_{f e}^{T}\left(V_{P N}^{T} V_{P N}+\Lambda_{N}^{2}\right) U_{f e}+\Lambda_{K}^{2}+\Lambda_{D}^{2}\right)^{-1}\left(P_{c N} \tilde{D}_{P t+k, N}+\psi(t)\right)
$$

Also recall from (41) and (45),

$$
\tilde{D}_{P t+k, N}^{0}=P_{C N} \tilde{D}_{P t+k, N}=P_{C N}\left(D_{P t+k, N}+C_{P N} A_{N} \hat{x}(t+k \mid t)\right)
$$

Thus, the optimal gains in (49) can be simplified further as follows:

$$
k_{c}(t)=-X_{N}^{-1}\left(\tilde{D}_{P t+k, N}^{0}+\psi(t)\right)
$$

where

$$
\tilde{D}_{P t+k, N}^{0}=P_{C N} D_{P t+k, N}+C_{\phi} \hat{x}(t+k \mid t)
$$

\section{Asymptotic behaviour}

Observe from (49) that if $\Lambda_{D}^{2} \rightarrow \infty \times I$ the limiting gain $k_{c}(t)=k_{c}(t-1)$ and the gains become constant. Similarly, if $\Lambda_{K}^{2} \rightarrow \infty \times I$ the limiting gain $k_{c}(t)=\bar{k}_{c}$ and the gains become equal to the constant initial PID gain settings.

\section{Minimum-cost}

Substituting in (47) for $\tilde{D}_{P t+k, N}^{0}=U_{f e}^{T} V_{P N}^{T} \tilde{D}_{P t+k, N}$, using (52) and substituting for the gain $k_{c}(t)$ in (51), the minimum-cost becomes:

$$
J_{\min }=\tilde{D}_{P t+k, N}^{T} \tilde{D}_{P t+k, N}-\left(\tilde{D}_{P t+k, N}^{0 T}+\psi(t)^{T}\right) X_{N}^{-1}\left(\tilde{D}_{P t+k, N}^{0}+\psi(t)\right)+\bar{J}_{0}
$$

where $\bar{J}_{0}=\bar{k}_{c}^{T} \Lambda_{K}^{2} \bar{k}_{c}+k_{c}^{T}(t-1) \Lambda_{D}^{2} k_{c}(t-1)+J_{0}$. The $\bar{k}_{c}$ is fixed and $k_{c}(t-1)$ is also known. 


\section{Theorem 1: $\quad$ Restricted Structure-Generalized Predictive Controller}

Consider the linear system and assumptions introduced in §2, where the sub-system $\mathcal{W}_{1 \mathrm{k}}=I$. The restricted structure generalized predictive controller is required to minimize the following cost-index:

$$
J=E\left\{J_{t}\right\}=E\left\{E_{P t+k, N}^{T} E_{P t+k, N}+U_{t, N}^{0 T} \Lambda_{N}^{2} U_{t, N}^{0}+\tilde{k}_{c}^{T}(t) \Lambda_{K}^{2} \tilde{k}_{c}(t)+\Delta \tilde{k}_{c}^{T}(t) \Lambda_{D}^{2} \Delta \tilde{k}_{c}(t) \mid t\right\}
$$

The RS-GPC controller can be implemented as follows:

$$
u(t)=L_{u}\left(z^{-1}\right) \sum_{j=1}^{N_{e}} f_{j}\left(z^{-1}, k_{j}(t)\right) e_{L}(t)=L_{u}\left(z^{-1}\right) F_{e}(t) k_{c}(t)
$$

where the functions $f_{j}\left(z^{-1}, k_{j}(t)\right)$ for $j \in\left[1, N_{e}\right]$ are specified for the chosen $R S$ controller structure, and where $e_{L}(t)=L_{e}\left(z^{-1}\right) e_{0}(t)$. The block-diagonal matrix $F_{e}(t)$ has the form:

$$
F_{e}(t)=\operatorname{diag}\left\{e_{f 1}(t) \quad e_{f 2}(t) \quad \cdots \quad e_{f m}(t)\right\}
$$

where for each $i=\{1,2, \ldots, m\}$ the row vector $e_{f i}(t)=\left[\begin{array}{llll}f_{e}^{i 1} & f_{e}^{i 2} & \cdots & f_{e}^{i r}\end{array}\right]$, and these functions are pre-specified by the designer. The optimal feedback controller gains are chosen to minimize (54). By invoking a form of the receding horizon philosophy, the $R S-G P C$ optimal time-varying gains satisfy:

$$
\begin{array}{r}
k_{c}(t)=-X_{N}^{-1}\left(\tilde{D}_{P t+k, N}^{0}-\Lambda_{K}^{2} \bar{k}_{c}-\Lambda_{D}^{2} k_{c}(t-1)\right) \\
=-X_{N}^{-1}\left(P_{C N} D_{P t+k, N}+C_{\phi} \hat{x}(t+k \mid t)+\psi(t)\right)
\end{array}
$$

where the matrices $\psi(t)=-\Lambda_{K}^{2} \bar{k}_{c}-\Lambda_{D}^{2} k_{c}(t-1), X_{N}=U_{f e}^{T}\left(V_{P N}^{T} V_{P N}+\Lambda_{N}^{2}\right) U_{f e}+\Lambda_{K}^{2}+\Lambda_{D}^{2}$, and $P_{c N}=U_{f e}^{T} V_{P N}^{T}$ and $C_{\phi}=U_{f e}^{T} V_{P N}^{T} C_{P N} A_{N}$. The total gain vector follows: 


$$
k_{c}=\left[\begin{array}{llll}
k_{c 1}^{T} & k_{c 2}^{T} & \cdots & k_{c m}^{T}
\end{array}\right]=\left[\begin{array}{llll}
\underbrace{k_{c}^{11 T} k_{c}^{12 T} \cdots k_{c}^{1 r T}}_{\text {channel 1 gains }} & \underbrace{k_{c}^{21 T} k_{c}^{22 T} \cdots k_{c}^{2 r T}}_{\text {channel } 2 \text { gains }} & \cdots & \underbrace{k_{c}^{m 1 T} k_{c}^{m 2 T} \cdots k_{c}^{m r T}}_{\text {channel } \text { gains }}
\end{array}\right]^{T}
$$

The vector of future controls may be obtained as:

$$
U_{t, N}=U_{f e}(t) k_{c}(t)
$$

where $U_{f e}^{T}(t)=\left[\begin{array}{llll}\left(L_{u}\left(z^{-1}\right) F_{e}(t)\right)^{T} & \left(L_{u}\left(z^{-1}\right) F_{e}(t+1)\right)^{T} \quad \cdots \quad\left(L_{u}\left(z^{-1}\right) F_{e}(t+N)\right)^{T}\end{array}\right]^{T}$

Solution: The RS-GPC proof follows by collecting the results above.

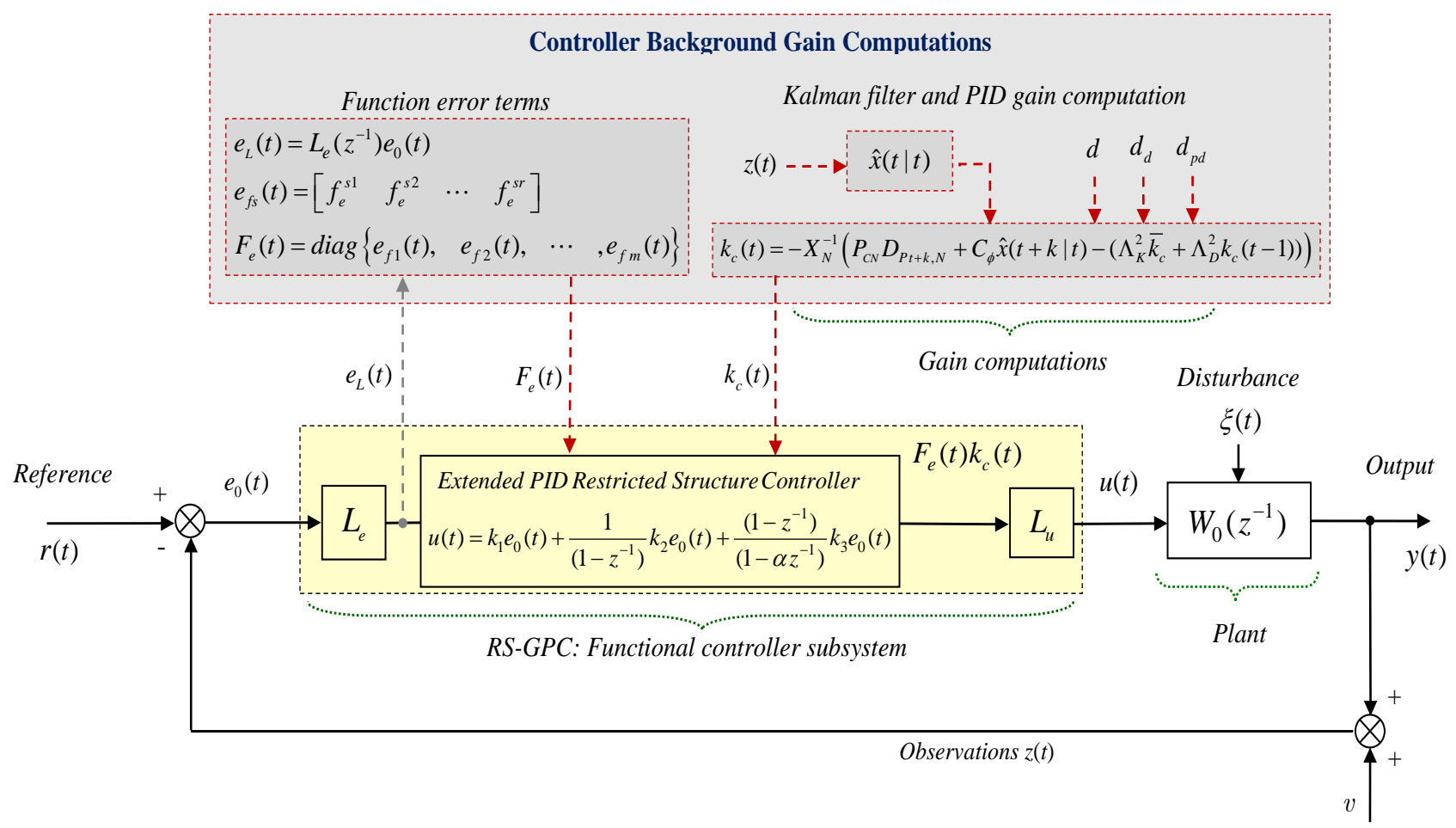

Figure 4. RS-GPC State-Space Controller Structure

\section{Comments on the Form of the solution}

The $R S$ optimal control problem is illustrated in Fig. 4. The controller is parameterized so that it has a conventional cascade form and the gains are found to minimize the costfunction (36). The PID structure in Fig. 4 is only representative of possible $R S$ control 
solutions. The theory applies for any $R S$ controller, which can be represented by a summation of transfer-function terms multiplied by gains.

\section{Numerical robustness}

The solution for the RS-GPC optimal control (57) depends upon the inverse of $X_{N}$. This time-varying matrix is full-rank because of the cost-weighting definitions. The expression for the gain-vector is similar to the vector of future controls in the usual GPC solution. However, the denominator matrix in (57) will often be of lower dimension. The weightings $\Lambda_{K}^{2}$ and $\Lambda_{D}^{2}$ depend on the number of the $R S$-controller gains and they ensure that $X_{N}$ does not become singular. The gains are not penalized in the cost-functions of traditional model predictive controls. However, it is valuable to be able to cost and tune these gains, and avoid numerical problems with near singular $X_{N}$.

\subsection{Square of Sum Optimization Problem}

The problem considered here is a special cost-minimization control problem, which is needed to motivate a nonlinear predictive control problem introduced later. The solution is obtained by completing the squares in Appendix 4.

\section{Theorem 2: $\quad$ Equivalent Cost-Minimization Problem}

Consider the system and assumptions introduced in §2, where the input subsystem $\mathcal{W}_{1 \mathrm{k}}=I$ and the minimization of the RS-GPC cost-index (36), where the vector of optimal RS-GPC controls is given by (51). Let a multi-step cost-index be defined as follows:

$$
\begin{gathered}
\tilde{J}(t)=E\left\{\Phi_{P t+k, N}^{T} \Phi_{P t+k, N} \mid t\right\} \\
\Phi_{P t+k, N}=P_{C N} E_{P t+k, N}+F_{C N}^{0} U_{t, N}^{0}+F_{C N}^{1} \tilde{k}_{c}(t)+F_{C N}^{2} \Delta \tilde{k}_{c}(t)
\end{gathered}
$$


Let the weightings $P_{C N}=U_{f e}^{T} V_{P N}^{T}, F_{C N}^{0}=U_{f e}^{T} \Lambda_{N}^{2}, F_{C N}^{1}=\Lambda_{K}^{2}, F_{C N}^{2}=\Lambda_{D}^{2}$ and $V_{P N}=C_{P N} B_{N}+E_{P N}$, and define $X_{N}=U_{f e}^{T}\left(V_{P N}^{T} V_{P N}+\Lambda_{N}^{2}\right) U_{f e}+\Lambda_{K}^{2}+\Lambda_{D}^{2}$. Then the vector of optimal gains becomes:

$$
k_{c}(t)=-X_{N}^{-1}\left(P_{C N} D_{P t+k, N}+C_{\phi} \hat{x}(t+k \mid t)-\Lambda_{K}^{2} \bar{k}_{c}-\Lambda_{D}^{2} k_{c}(t-1)\right)
$$

where $C_{\phi}=U_{f e}^{T} V_{P N}^{T} C_{P N} A_{N}$ and $\psi(t)=-\Lambda_{K}^{2} \bar{k}_{c}-\Lambda_{D}^{2} k_{c}(t-1)$. This expression for the gain vector is identical to the RS-GPC controller in (51) or Theorem 1. The optimal control can be realized as in shown in Fig. 5. The vector of future controls is given as follows:

$$
U_{t, N}=U_{f e}(t) k_{c}(t) \quad \text { or } \quad U_{t, N}=-U_{f e} X_{N}^{-1}\left(\tilde{D}_{P t+k, N}^{0}+\psi(t)\right)
$$

Solution: The proof follows by collecting results in Appendix 4.

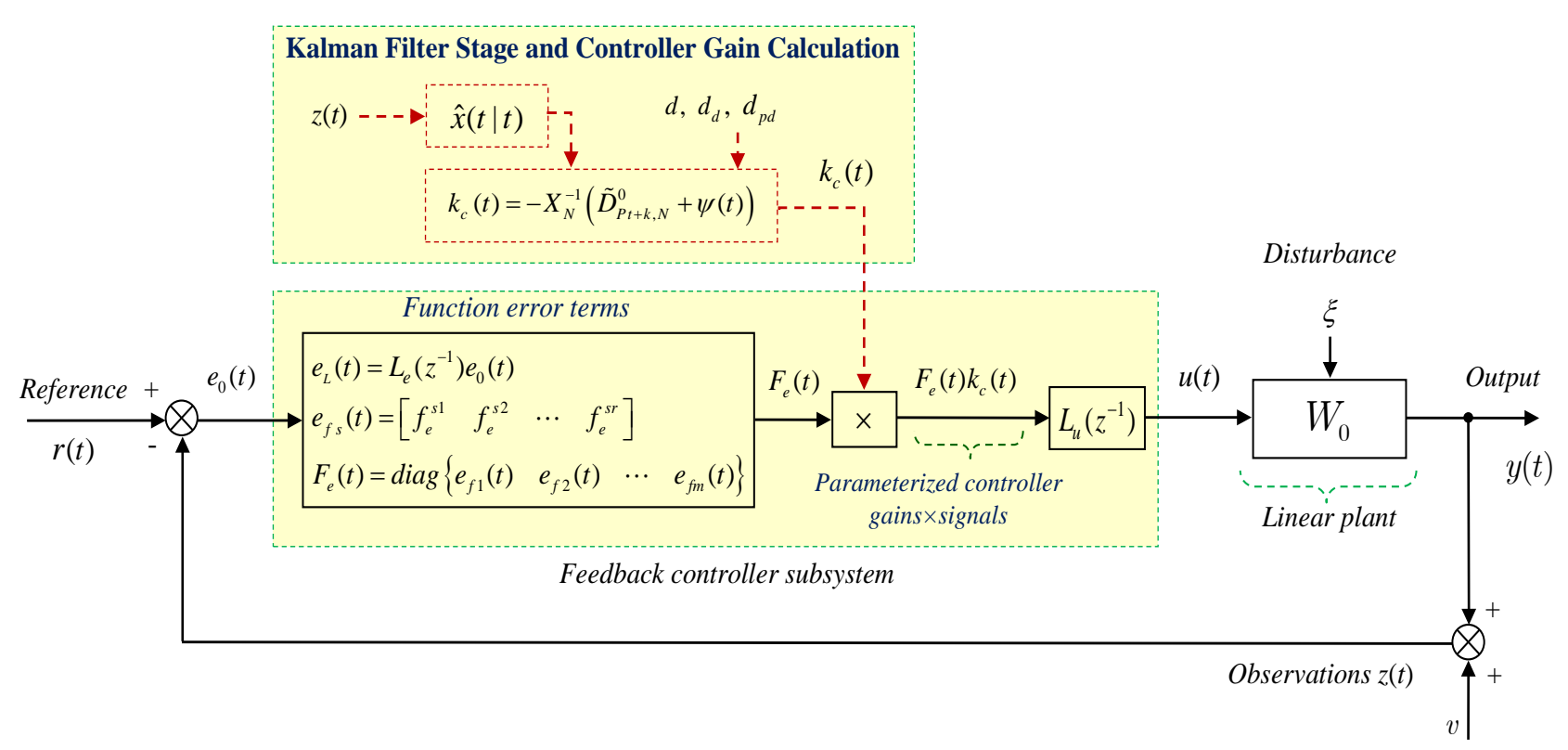

Figure 5. RS-GPC State-Space Controller Structure 


\subsection{Cost-Function Tuning Variables}

Retuning the controller should be simple. For a scalar problem, the weighting on the control signal provides a simple way to vary the speed of response of the system. The weighting on the error can be chosen to be unity. In this case, or if integral action is included, then the integrator weighting gain can be scaled to unity. The remaining weightings $F_{C N}^{1}=\Lambda_{K}^{2}$ and $F_{C N}^{2}=\Lambda_{D}^{2}$ are on the magnitude of the gains and rate of gain changes. The cost of control is a term introduced by Isaac Horowitz to drew attention to the cost of feedback. High gains have disadvantages and the ability to reduce these gains whilst not sacrificing performance is valuable, providing the control action is satisfactory.

\subsection{Stability}

The fact that the solution provides an optimal control does not of course guarantee stability. If the system has no disturbance or reference changes, then from (62), the RScontroller gains become constant and the stability conditions are those for a linear timeinvariant system, and the characteristic polynomial can be inspected. Under more general changing conditions, the system is time-varying. Nevertheless, if the rate of change of gains is controlled and the gains vary sufficiently slowly, it should be possible to establish stability conditions using similar analysis to that for adaptive systems. However, if hard constraints on controller gains are applied the region of operation is well defined. The approach of Diaz-Rodriguez and Bhattacharyya (2016) defines a stabilizing set of PI (or PID) controllers for such systems. The definition of the cost-function weightings is important since they determine performance and stability (illustrated in the example).

\section{SI Automotive Engine Design}

A spark-ignition (SI) engine simulation model was used to evaluate the design approach, and for simplicity the engine control model provided in Matlab/Simulink was utilized. The model consists of the simplified dynamics of the SI engine air path, as well as a simple vehicle dynamics model with inputs and outputs shown in Fig. 6. 


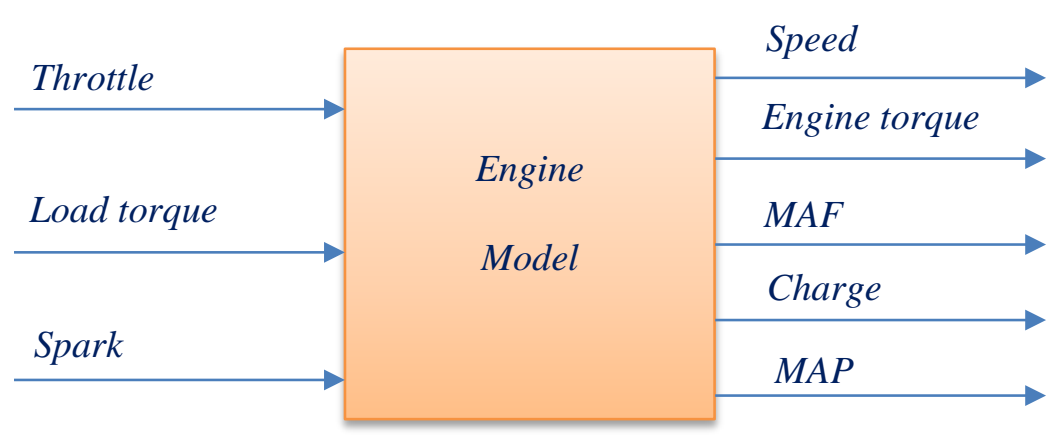

Figure 6. Engine Model: Inputs, Outputs and Parameters

The original control problem involved engine idle speed control by manipulating the throttle input, subject to a varying load torque. The control objective here is to keep the engine speed at the set-point irrespective of the load torque disturbance. For comparison purposes, a PI-controller with an anti-windup mechanism is included. A speed set-point signal (in rpm) is to be tracked, in the presence of load torque. The controller computes the necessary throttle angle based on the desired rpm and the measured engine speed, which is the output of the vehicle dynamics subsystem.

\section{Model Equations}

The equations for various engine model subsystems follow. The signals under consideration are as follows:

- controlled output: engine speed $N(\mathrm{rpm})$

- measurements: engine speed and intake manifold pressure $P_{i m}(b a r)$

- control input: throttle angle $\theta$ (deg.)

- unknown varying disturbance input: load torque $T_{\text {load }}(\mathrm{Nm})$

- known input: spark advance $S A$ (deg.).

Throttle flow: $\dot{m}_{a}=\operatorname{sgn}_{f} \cdot f(\theta) \cdot \Psi\left(p_{r}\right)$ where $\operatorname{sgn}_{f}=\left\{\begin{array}{cc}1, & P_{a}>P_{m} \\ 0, & P_{a}=P_{m} \\ -1, & P_{a}<P_{m}\end{array} \quad\right.$ (flow direction) 


$$
\begin{aligned}
& f(\theta)=2.821-0.05231 \cdot \theta+0.10299 \cdot \theta^{2}-0.00063 \cdot \theta^{3} \quad \text { (discharge coefficient) } \\
& p_{r}=\min \left(\left(P_{m} / P_{a}\right),\left(P_{a} / P_{m}\right)\right) \quad \text { (pressure ratio) } \\
& \Psi\left(p_{r}\right)=\left\{\begin{array}{cc}
2 \sqrt{p_{r}\left(1-p_{r}\right)}, & p_{r} \geq 0.5 \\
1.0 & p_{r}<0.5 \quad \text { (sonic flow) }
\end{array} \text { and atmospheric pressure is set to } \mathrm{P}_{\mathrm{a}}=1\right. \text { bar. }
\end{aligned}
$$

Intake manifold pressure: $\dot{P}_{m}=\frac{R T}{V_{m}}\left(\dot{m}_{a}-f_{\text {pump }}\left(P_{m}, N\right)\right)$ where $R T / V_{m}=0.41328$ and

$$
f_{\text {pump }}\left(P_{m}, N\right)=-0.366+0.08979 \cdot P_{m} \cdot N-0.0337 \cdot N \cdot P_{m}^{2}+0.0001 \cdot P_{m} \cdot N^{2} \quad \text { (pumping) }
$$

Air charge: $C A C=-0.0001 \cdot N+0.1812 \cdot P_{m}-0.0725 \cdot P_{m}^{2}+0.0005 \cdot P_{m} \cdot N+0.0362$

Power stroke delay: This parameter defines the variable time delay affecting the air charge delivered for combustion, $t_{d e l}=\pi / N$.

Engine torque: $T_{\text {eng }}=f_{T Q}(C A C, C F C, S A, N)$ where,

$$
\begin{aligned}
& f_{\mathrm{TQ}}(C A C, A F R, S A, N)=-181.3+379.36 \cdot C A C+21.91 \cdot A F R-0.85 \cdot A F R^{2}+0.26 \cdot S A+ \\
& \quad-0.0028 \cdot S A^{2}+0.027 \cdot N-0.000107 \cdot N^{2}+0.00048 \cdot N \cdot S A+2.55 \cdot S A \cdot C A C-0.05 \cdot S A^{2} \cdot C A C
\end{aligned}
$$

The stoichiometric air-fuel ratio $(A F R)$ is assumed $A F R=14.6$.

Vehicle dynamics: $\dot{N}=(1 / J)\left(T_{\text {eng }}-T_{\text {load }}\right)$, where the vehicle inertia $J=0.14$.

\subsection{Design Aspects}

The control problem is to manipulate the throttle angle to track the engine speed set-point subject to unknown drag/load torque variations. The test scenarios involve speed set-point step changes from 2000 to $2500 \mathrm{rpm}$, and load torque varying between 20 to $25 \mathrm{Nm}$. Let the prediction-horizon $N=20$ and delay $k=1$. The cost weightings:

$$
\begin{aligned}
& \Lambda_{N}^{2}=400 . \mathrm{I}, \quad \Lambda_{D}^{2}=1 \mathrm{e}^{10} \times \operatorname{diag}\left\{\begin{array}{lll}
1 & 0.1 & 1
\end{array}\right\}, \quad \Lambda_{K}^{2}=1 \mathrm{e}^{9} \times \operatorname{diag}\left\{\begin{array}{lll}
12 & 1 & 0.1
\end{array}\right\}, \\
& P_{c}=0.25 \times\left(1-0.98 \mathrm{z}^{-1}\right) /\left(1-\mathrm{z}^{-1}\right) \text { and } \mathcal{F}_{\mathrm{ck}}\left(z^{-1}\right)=\left(100-30 z^{-1}\right) /\left(1-0.1 z^{-1}\right) .
\end{aligned}
$$


The frequency response of the weighting on control is shown in Fig. 7 and the error weighting multiplied by the plant transfer function (between throttle angle (degrees) and speed (rpm)). The plots cross at about 2 radians per second and the rule of thumb is that the bandwidth should be in the region of 0.5 seconds.

The step-responses shown in Fig. 8 are for the two $R S$ cases of using absolute gains or gain deviations, and final response is for the use of MPC. In this latter case a Generalized Predictive Control algorithm was used as in Ordys and Clarke (1993). None of the methods is clearly preferable, since it is likely similar results can be obtained by different weightings. This does not apply to the constrained gain cases where the particular problem may dictate the best choice. The gains in Fig. 9 indicate the gains only change when disturbances or reference changes occur.

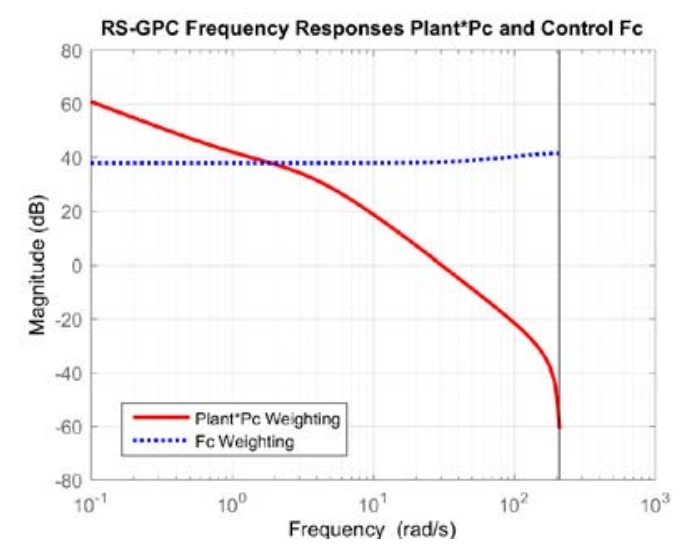

Figure 7. Weighting Frequency Responses Plant $\times \mathrm{P}_{\mathrm{C}}$ and $\mathrm{F}_{\mathrm{C}}$

\section{Comparison with Fixed PID}

The results shown in Fig. 10 compare the absolute gain case against a traditional PID solution. The torque control is clearly superior, but it is more the advantages of the model based solution for $R S-G P C$ which is the main benefit. 

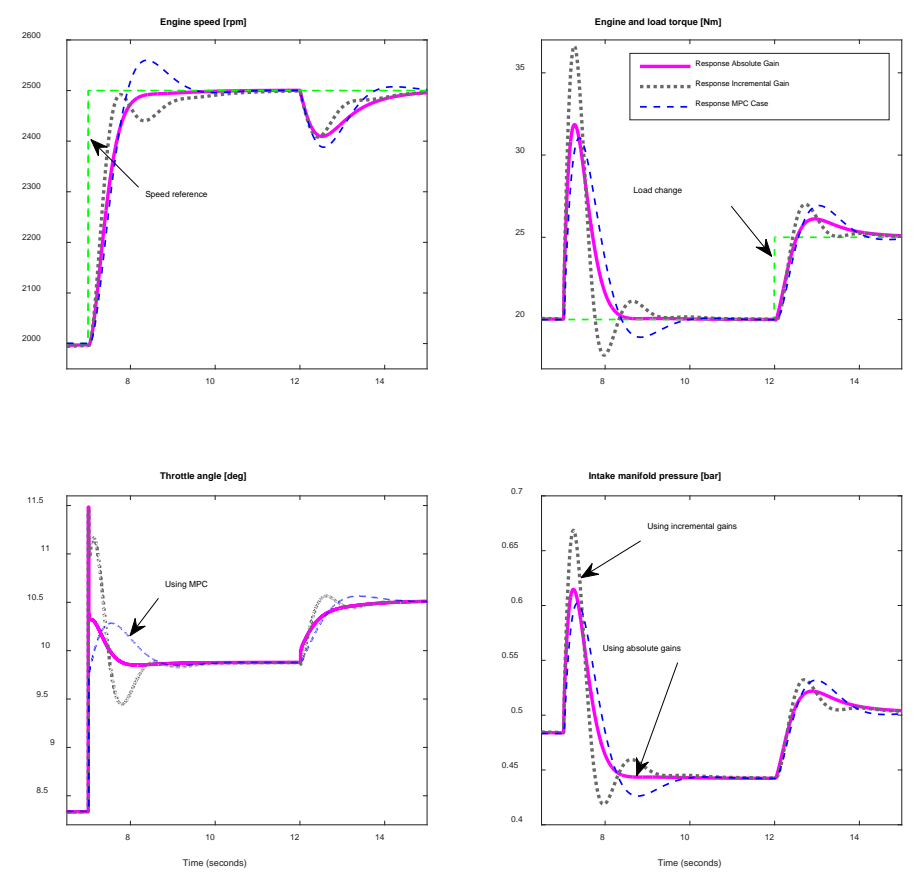

Figure 8. Comparison of Time Responses for RS-Absolute and Gain Deviation Cases, and MPC Design (No Reference Knowledge, Gain Constraint, Rate of Change Weight)
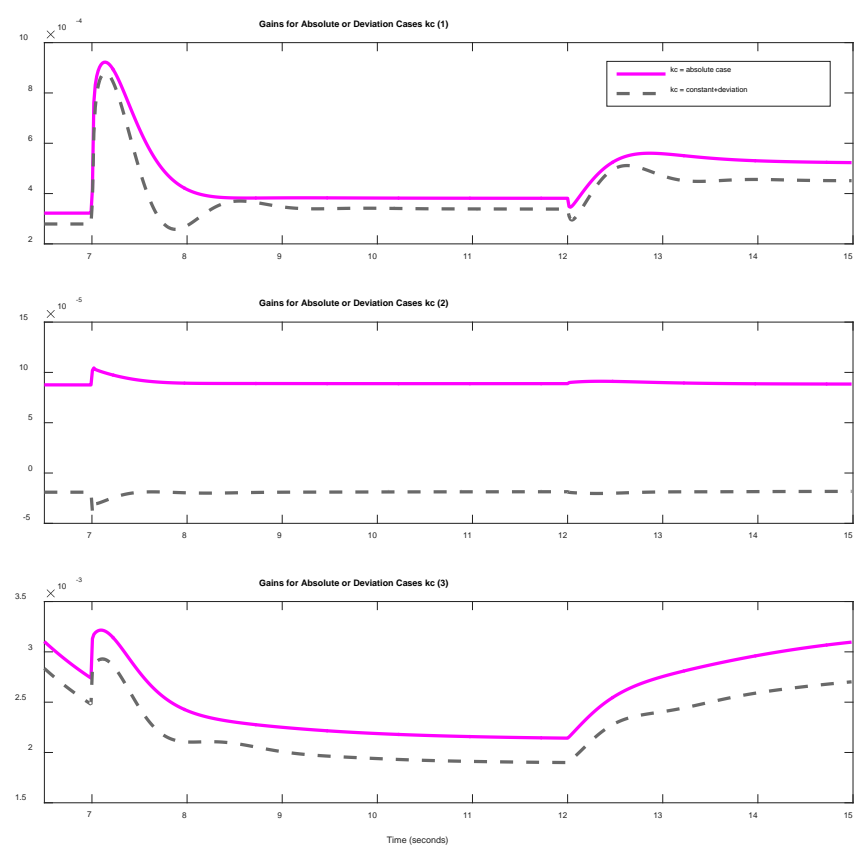

Figure 9. Comparison of Responses of Gains for Absolute and Gain Deviation Cases 

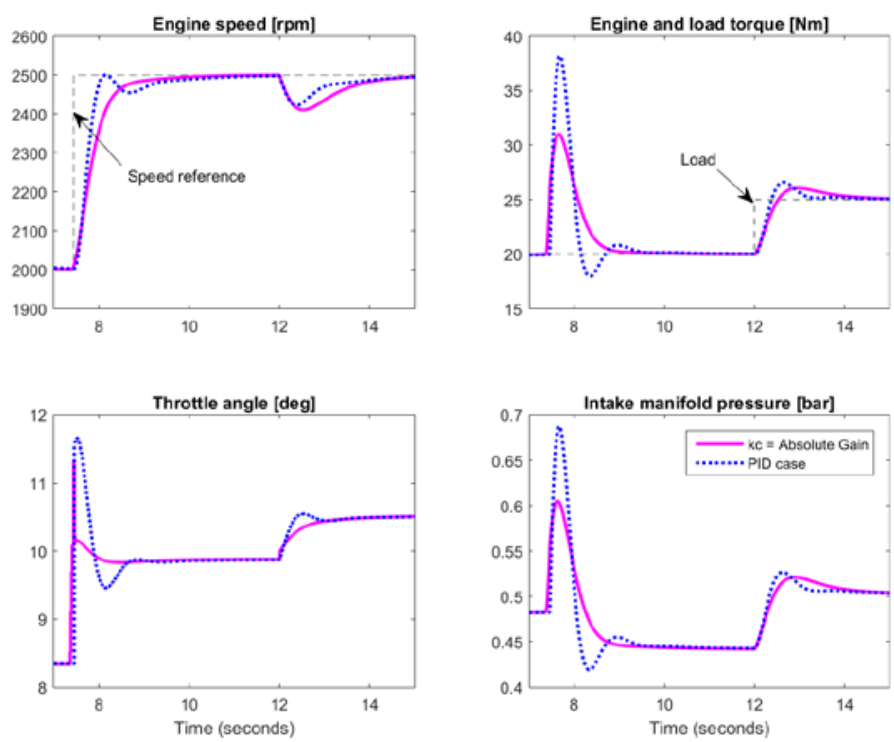

Figure 10. Comparison with PID Including of RS-GPC with Absolute Gains

\section{Effect of gain constraints}

For the case of constrained gain magnitudes let the constraints be set as: $k_{\max }=\left[\begin{array}{ll}2 \mathrm{e}-4 & 2 \mathrm{e}-\end{array}\right.$

$35 \mathrm{e}-3]^{\mathrm{T}}, k_{\min }=\left[\begin{array}{lll}0 & 0.5 \mathrm{e}-5 & 1 \mathrm{e}-5\end{array}\right]^{\mathrm{T}}, \Delta k_{\max }=\left[\begin{array}{lll}1 & 1 & 1\end{array}\right]^{\mathrm{T}}, \quad \Delta k_{\min }=\left[\begin{array}{lll}-1 & -1 & -1\end{array}\right]^{\mathrm{T}} . \mathrm{A}$ comparison of the time-responses for the unconstrained and constrained cases using absolute gains are shown in Fig. 11. The gains in Fig. 12, show the constraints are active.
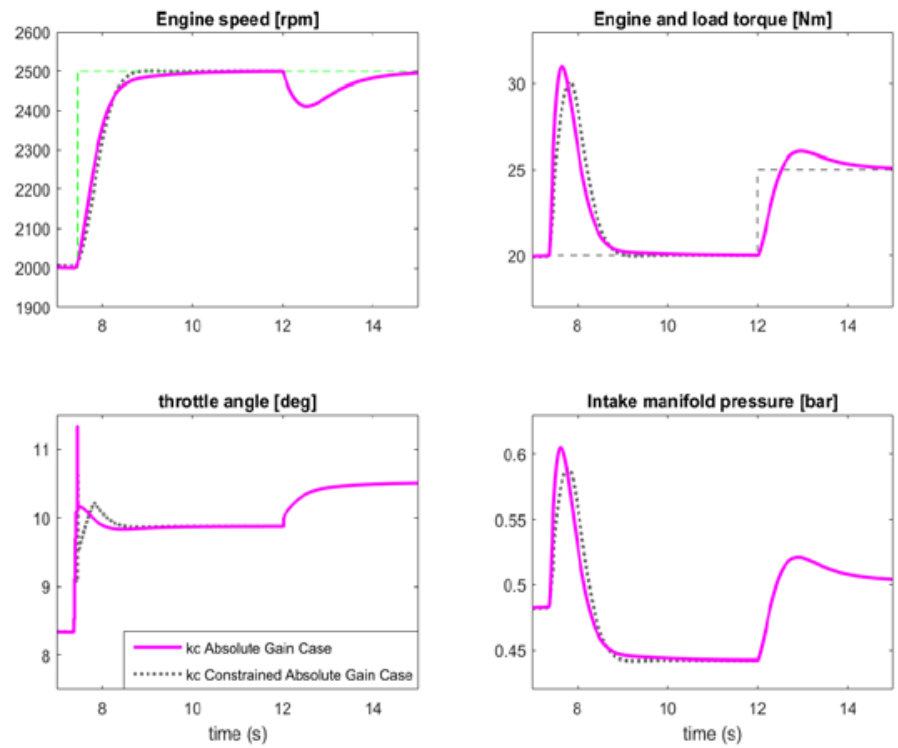

Figure 11. Absolute Gain Case Showing Responses for Unconstrained and Constrained

Gain Cases (Includes No Future Reference Knowledge, Rate of Change Weighting) 

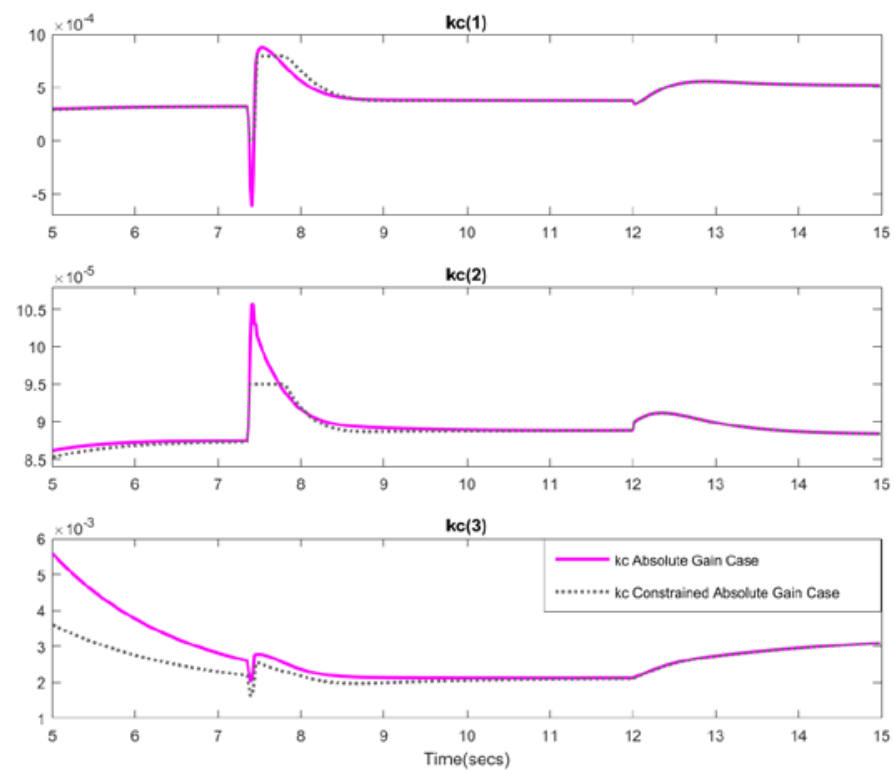

Figure 12. Absolute Gain Responses for Unconstrained and Constrained Cases

\section{Robustness}

The plant model is mismatched by inserting a mismatch transfer-function on each of the plant state signal paths where the mismatch was defined as:

$$
\begin{aligned}
& W_{\text {mismatch }}(1,1)=157.9 /\left(s^{2}+6.283 s+157.9\right) \\
& W_{\text {mismatch }}(2,2)=268.5 /\left(s^{2}+7.54 s+157.9\right)
\end{aligned}
$$

Clearly, the PID controller response, shown in Fig. 13 is more sensitive than the design using absolute gains (compare with PID in Fig. 10). The RS-GPC controller does of course adapt in some sense, since the computed restricted structure controller PID gains are modified when mismatch is present as shown in Fig. 14. Not surprisingly, there is more gain variation needed to cope with the mismatch case.

\section{Transport delay}

The RS-GPC absolute gain method and PID control are compared in Fig. 15 when an additional 10-step delay uncertainty is introduced in the plant. Mismatch on the transport- 
delay can be very destabilizing and the PID controller responses deteriorate due to the addition of the transport delay elements. The RS-GPC design is not phased too much by this error in knowledge of the delay. It is the optimised gains from the predictive control, shown in Fig. 16, which compensates for the mismatch.
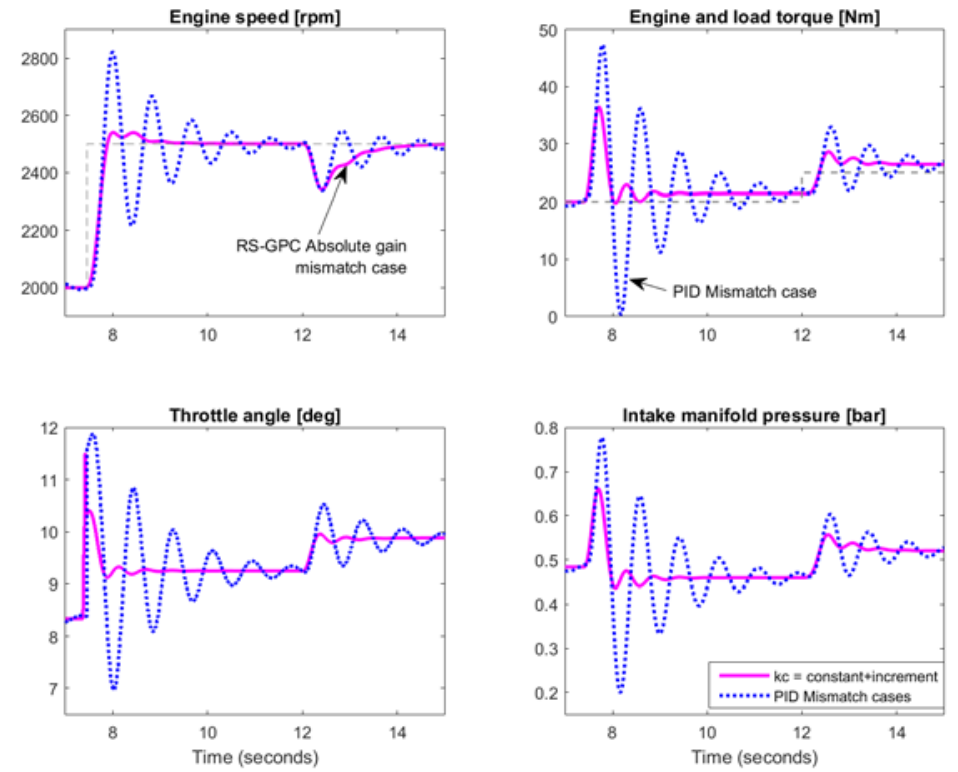

Figure 13. Comparison with PID of RS-GPC with Absolute Gains for Mismatch Plant Model (Includes No Future Reference, Gain Constraint, Rate of Change Weighting)
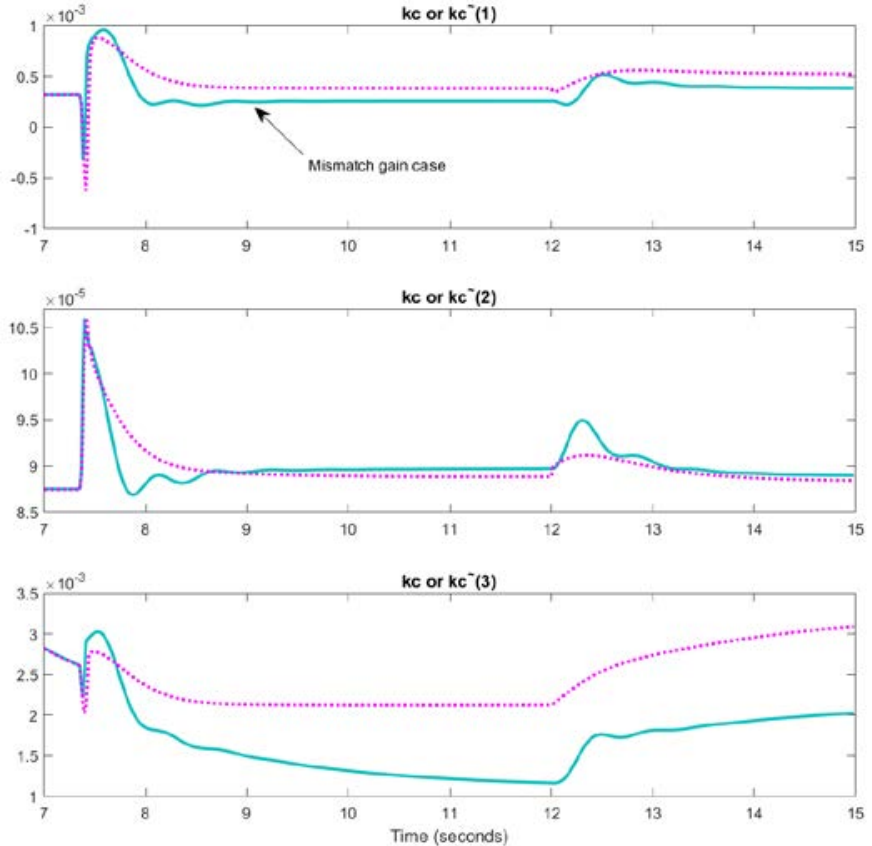

Figure 14. Absolute Gains of RS-GPC for Mismatch and No Mismatch Cases 

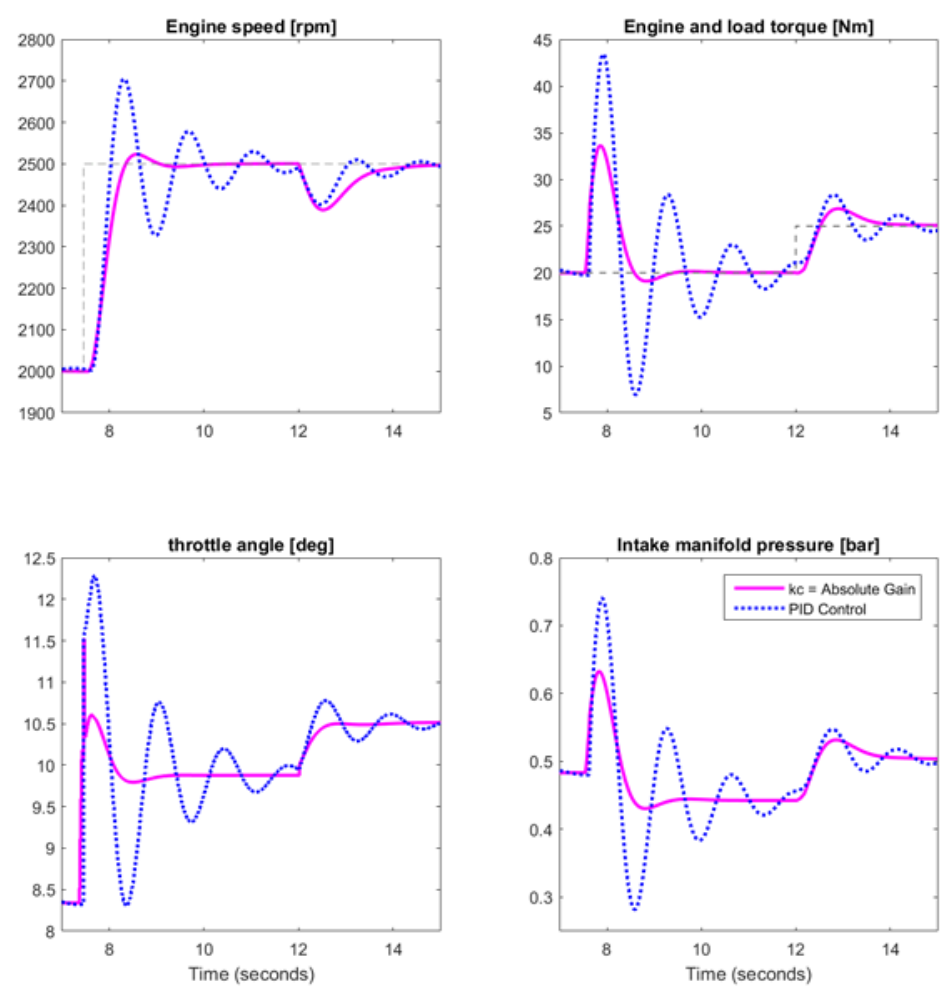

Figure 15. Comparison Responses for Mismatch on Delay of 10 Steps RS-GPC Absolute Gain Case and PID
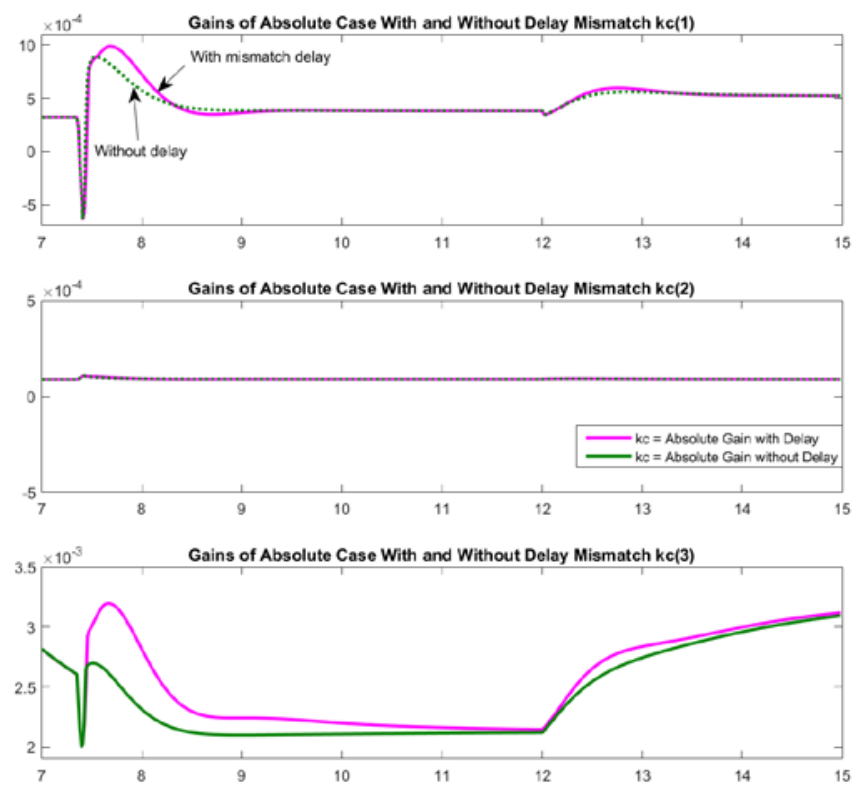

Figure 16. Comparison Gains for RS-GPC Absolute Gain Case with and without Mismatch on Delay of 10 Steps 

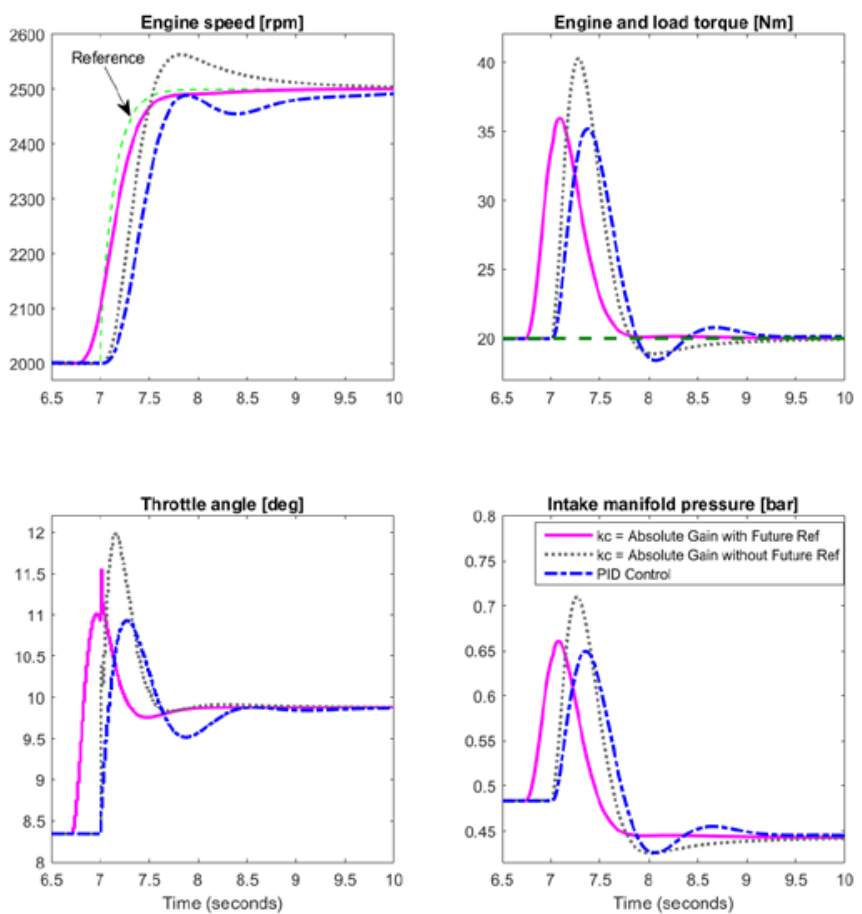

Figure 17. Gain Responses for RS-GPC Absolute Gain Case with Reference Model, with and without Future Reference Compared to PID (Including No Gain Constraint)

\section{Future setpoint knowledge}

The RS-GPC controller provides a predictive capability, as shown in the Fig. 17 . This applies to both the absolute and incremental gain cases, however, in this case the absolute gain formulation has the advantage since a larger portion of the gains are allowed to vary. A reference model $W_{w}\left(z^{-1}\right)$ was also used in this case and the gain of $P_{c}$ was increased. The results are particularly good for the absolute gain case with the shaped future reference signal.

\section{Changing Gains by Modifying Functions Parameterizing Controller}

If the functions that define the controller structure $f_{j}\left(z^{-1}, k_{j}(t)\right)$ are modified by using scalar multipliers, the optimal solution should change. If the functions $f_{j}\left(z^{-1}, k_{j}(t)\right)$, 
defined in (26), are multiplied by scalars $(\alpha, \beta, \gamma)$ they can influence the proportional, integral and derivative behaviour. Recall the criterion:

$$
J=E\left\{J_{t}\right\}=E\left\{E_{P t+k, N}^{T} E_{P t+k, N}+U_{t, N}^{0 T} \Lambda_{N}^{2} U_{t, N}^{0}+\tilde{k}_{c}^{T}(t) \Lambda_{K}^{2} \tilde{k}_{c}(t)+\Delta \tilde{k}_{c}^{T}(t) \Lambda_{D}^{2} \Delta \tilde{k}_{c}(t) \mid t\right\}
$$

and note from (57):

$$
k_{c}(t)=-X_{N}^{-1}\left(U_{f e}^{T} V_{P N}^{T} D_{P t+k, N}+U_{f e}^{T} V_{P N}^{T} C_{P N} A_{N} \hat{x}(t+k \mid t)-\Lambda_{K}^{2} \bar{k}_{c}-\Lambda_{D}^{2} k_{c}(t-1)\right)
$$

and $X_{N}=U_{f e}^{T}\left(V_{P N}^{T} V_{P N}+\Lambda_{N}^{2}\right) U_{f e}+\Lambda_{K}^{2}+\Lambda_{D}^{2}$. The behaviour in the deviation gain case, follows from inspection of these equations. In this case $k_{c}(t)=\bar{k}_{c}+\tilde{k}_{c}(t)$ and the cost terms involving $\Lambda_{K}^{2}+\Lambda_{D}^{2}$ are quite dominant, since they act on gain deviations, and much of the gains are fixed at $\bar{k}_{c}$. Now a scalar multiplication of the functions affects the $U_{f e}^{T}(t)$. The consequence is that an increase in the function gains increases the gain $k_{c}(t)$. This is shown in the deviation in gains case in Fig. 18.
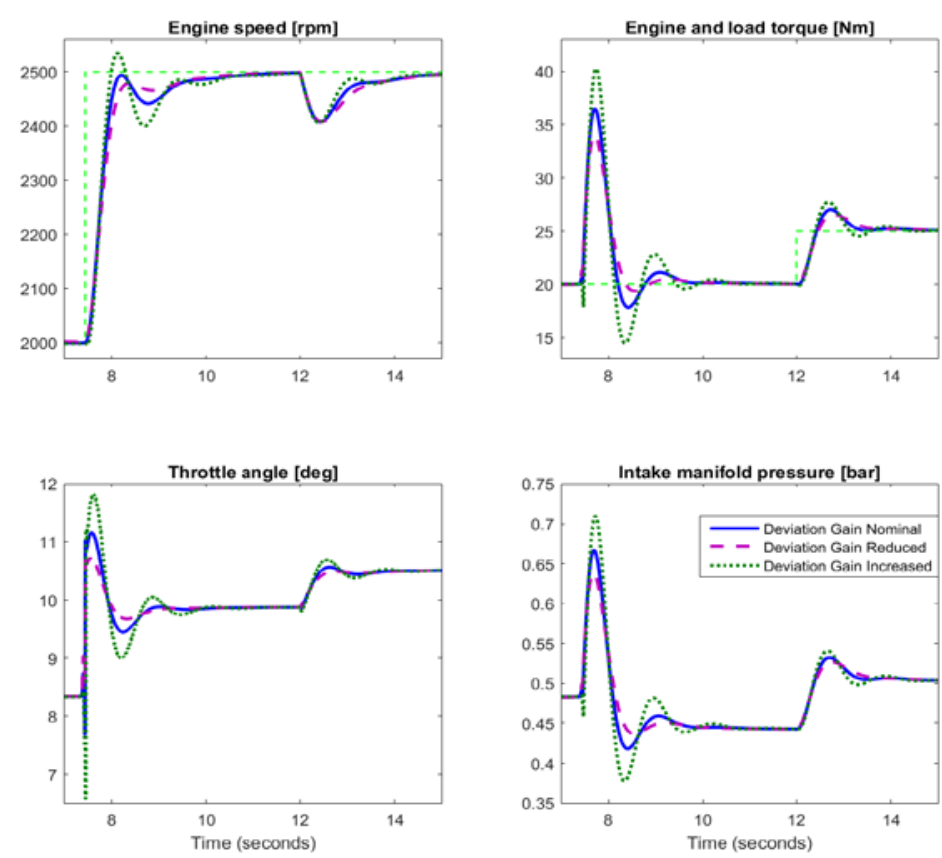

Figure 18. Comparison Responses for RS-GPC Gain Deviation Case Nominal, Lower and Higher Gains in Functions (Including No Future Setpoint, Gain Constraint) 

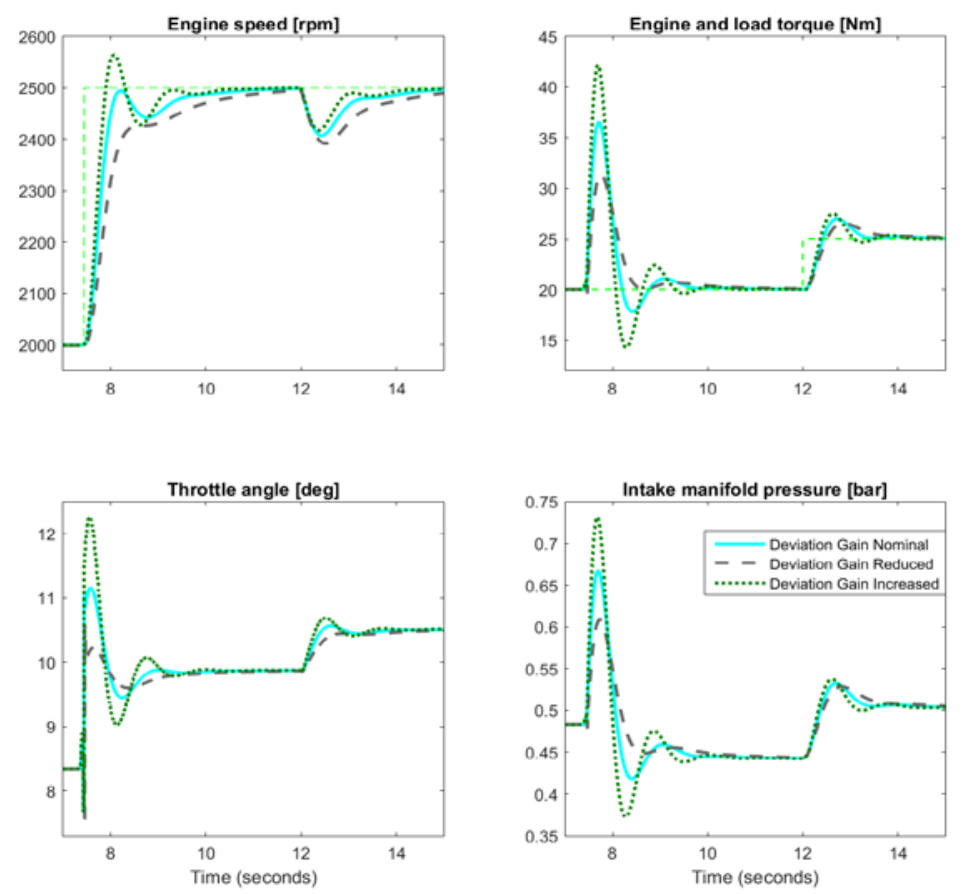

Figure 19. Responses for RS-GPC Deviation Gain Case Nominal, Lower and Arbitrary Higher Gains (Including No Future Setpoint, No Gain Constraint)

If the PID gains are changed directly in an arbitrary manner, by simply multiplying the gain vector elements in $k_{c}(t)$ by scaling factors, the results are as shown in Fig. 19 for the deviation gain cases. The results are not optimal and changes are quite arbitrary, but it does provide a mechanism for retuning a plant.

\subsection{Lessons from the Example}

The lessons learned may be summarized as:

- The use of time-varying gains can lead to better responses in comparison with classical fixed-gain controllers.

- Mismatch for the cases tried had a lot less effect when using the RS-GPC controller relative to a classical fixed gain PID controller. This also applied to mismatch in the knowledge of the transport delay. 
- The controller gains only change when the disturbance or reference changes.

- Arbitrary modification of the computed gains leads to predictable behaviour for simple modifications. For example, increasing or decreasing all gains by $20 \%$ speeds up or slows down responses correspondingly.

- The future reference information changes the time-varying control gains to improve responses but the mechanism is not as direct as traditional MPC.

\subsection{Simplifications for Implementation}

If the sample rate for the $R S$-controller is the same for the feedback loop as for the background processing the execution time may not be very different to a conventional GPC design (assuming system is linear). This occurs because the Kalman estimator and the optimization computations involve similar types of matrix in the two problems. The main matrix to be inverted does of course depend upon the controller parameterization chosen. For the results shown in Fig. 8 the $R S-G P C$ simulation had an elapsed time of 12.3536 seconds and the MPC elapsed time was 9.8957 seconds. The former depends upon the number of gains to be computed ( 3 for PID) and the background processing and the latter depends on the control horizon.

A benefit of the $R S$-controller is that numerical savings can be made because of the structure of the controller. There is no need to update the gains at the same sample rate as the feedback loop RS-controller. Using a lower sampling rate for the background processing should significantly reduce the computational burden. Another possible simplification is to compute and store the $R S$-controller gains to be implemented using scheduling. The procedure could involve:

- The controller can be used in a simulation using standard driving cycles or using a form of Monte Carlo testing. 
- The gains computed by the $R S$ controller can be averaged over different zones of operation and values stored according to operating point (driving conditions).

The resulting implementation should be much more efficient than traditional MPC in terms of computing resources.

\section{RS-GPC Control Problem for Nonlinear System}

A similar problem is now considered but one that reveals the link with traditional transport-delay compensation methods and with NGMV control problems Grimble (2005), Grimble and Majecki (2010a and 2010b). The unstructured input sub-system $\mathcal{W}_{1 k}$ is included in this case. The model for $\mathcal{W}_{1 k}$ can be nonlinear and the model is assumed to be in "black-box" form. That is, the model equations need not be known, and it is only assumed the output of $\mathcal{W}_{1 k}$ can be calculated for a given input. The actual input to the system is the control signal $u(t)$, shown in Fig. 2, rather than the input to the input unstructured sub-system $u_{0}(t)=\left(\mathcal{W}_{1 \mathrm{k}} u\right)(t)$.

The cost-function for the problem of interest may include an additional control signal costing term. If the smallest delay in each output channel of the plant is of magnitude $k$-steps this implies that the control signal $t$ affects the output at least $k$-steps later. For this reason, the dynamic control signal costing is defined to have the following form:

$$
\left(\mathcal{F}_{\mathrm{c}} u\right)(t)=z^{-k}\left(\mathcal{F}_{\mathrm{ck}} u\right)(t)
$$

The control weighting operator $\mathcal{F}_{c k}$ will be assumed to be full-rank and invertible and can be nonlinear. In analogy with the previous RS-GPC problem a multi-step cost-index may 
now be defined that is an extension of the cost-function in (91). Let the function $\mathcal{F}_{\mathrm{ck}, N} U_{t, N}$ be defined to have the simple diagonal form:

$$
\left(\mathcal{F}_{c k, N} U_{t, N}\right)=\operatorname{diag}\left\{\left(\mathcal{F}_{c k} u\right)(t),\left(\mathcal{F}_{c k} u\right)(t+1), \ldots,\left(\mathcal{F}_{c k} u\right)(t+N)\right\}
$$

Recall the parameterization $U_{t, N}=U_{f e}(t) k_{c}(t)$, so that the vector of inputs may be expressed as follows:

$$
U_{t, N}^{0}=\mathcal{W}_{1 k, N} U_{f e}(t) k_{c}(t)
$$

where $\left(\mathcal{W}_{1 k, N} U_{t, N}\right)=\operatorname{diag}\left\{\mathcal{W}_{1 k}, \mathcal{W}_{1 k}, \ldots, \mathcal{W}_{1 k}\right\} U_{t, N}=\left[\left(\mathcal{W}_{1 k} u\right)(t)^{T}, \ldots,\left(\mathcal{W}_{1 k} u\right)(t+N)^{T}\right]^{T}$

Extended Cost-Index: $\quad J_{p}=E\left\{\Phi_{P t+k, N}^{0 T} \Phi_{P t+k, N}^{0} \mid t\right\}$

where $\Phi_{P t+k, N}^{0}$ in (67) is an extension of (61), to include future control costing terms:

$$
\Phi_{P t+k, N}^{0}=P_{C N} E_{P t+k, N}+F_{C N}^{0} U_{t, N}^{0}+F_{c N}^{1} \tilde{k}_{c}(t)+F_{c N}^{2} \Delta \tilde{k}_{c}(t)+U_{f e}^{T} \mathcal{F}_{c k, N} U_{t, N}
$$

Recall $F_{c N}^{0}=U_{f e}^{T} \Lambda_{N}^{2}, F_{C N}^{1}=\Lambda_{K}^{2}, F_{C N}^{2}=\Lambda_{D}^{2}, U_{t, N}=U_{f e}(t) k_{c}(t), \tilde{k}_{c}(t)=k_{c}(t)-\bar{k}_{c}$ and $\Delta k_{c}(t)=k_{c}(t)-k_{c}(t-1)$. Hence, obtain:

$$
\begin{aligned}
& \Phi_{P t+k, N}^{0}=P_{C N} E_{P t+k, N}+U_{f e}^{T} \Lambda_{N}^{2} U_{t, N}^{0}+\Lambda_{K}^{2}\left(k_{c}(t)-\bar{k}_{c}\right)+\Lambda_{D}^{2}\left(k_{c}(t)-k_{c}(t-1)\right)+U_{f e}^{T} \mathcal{F}_{\mathrm{ck,N}} U_{t, N} \\
& =P_{C N} E_{P t+k, N}+\left(U_{f e}^{T} \Lambda_{N}^{2} \mathcal{W}_{1 k, N} U_{f e}+\Lambda_{K}^{2}+\Lambda_{D}^{2}+U_{f e}^{T} \mathcal{F}_{\mathrm{Ck}, N} U_{f e}\right) k_{c}(t)-\Lambda_{K}^{2} \bar{k}_{c}-\Lambda_{D}^{2} k_{c}(t-1)
\end{aligned}
$$

\subsection{RS-GPC with Unstructured Block Control Solution}

Only a brief summary of the solution of this problem is provided since it follows from very similar steps to those in Appendix 4. Observe from (92) that $\Phi_{P t, N}^{0}=\Phi_{P t, N}+z^{-k} U_{f e}^{T} \mathcal{F}_{c k, N} U_{t, N}$ and $\Phi_{P t+k, N}^{0}=\hat{\Phi}_{P t+k, N}^{0}+\tilde{\Phi}_{P t+k, N}^{0}$. Thence obtain,

$$
\hat{\Phi}_{P t+k, N}^{0}=P_{C N} \hat{E}_{P t+k, N}+\left(U_{f e}^{T} \Lambda_{N}^{2} \mathcal{W}_{1 k, N} U_{f e}+\Lambda_{K}^{2}+\Lambda_{D}^{2}+U_{f e}^{T} \mathcal{F}_{\mathrm{ck}, N} U_{f e}\right) k_{c}(t)+\psi(t)
$$


Substituting from (21) for the future predicted error $\hat{E}_{P t+k, N}$ :

$$
\begin{gathered}
\hat{\Phi}_{P t+k, N}^{0}=P_{C N} D_{P t+k, N}+P_{C N} C_{P N} A_{N} \hat{x}(t+k \mid t) \\
+\left(\Lambda_{K}^{2}+\Lambda_{D}^{2}+U_{f e}^{T} \mathcal{F}_{\mathrm{C} k, N} U_{f e}+\left(P_{C N} V_{P N}+U_{f e}^{T} \Lambda_{N}^{2}\right) \mathcal{W}_{1 k, N} U_{f e}\right) k_{c}(t)+\psi(t)
\end{gathered}
$$

The estimation error:

$$
\tilde{\Phi}_{P t+k, N}^{0}=\tilde{\Phi}_{P t+k, N}=U_{f e}^{T} V_{P N}^{T} \tilde{E}_{P t+k, N}
$$

The future predicted values of error in the signal $\hat{\Phi}_{P t+k, N}^{0}$, involves the estimated vector of weighted errors $\hat{E}_{P t+k, N}$ and these are orthogonal to $\tilde{E}_{P t+k, N}$. The estimation error is zero mean and the expected value of the product with a known signal is null, and hence the cost to be minimized may be written as follows:

$$
\tilde{J}(t)=\hat{\Phi}_{P t+k, N}^{0 T} \hat{\Phi}_{P t+k, N}^{0}+\tilde{J}_{1}(t)
$$

where the optimal control gives $\hat{\Phi}_{P t+k, N}^{0}=0$. The condition for optimality, that determines the optimal solution, noting $P_{c N}=U_{f e}^{T} V_{P N}^{T}$, becomes:

$$
\begin{gathered}
P_{c N} D_{P t+k, N}+P_{c N} C_{P N} A_{N} \hat{x}(t+k \mid t) \\
+\left(\Lambda_{K}^{2}+\Lambda_{D}^{2}+U_{f e}^{T} \mathcal{F}_{C k, N} U_{f e}+U_{f e}^{T}\left(V_{P N}^{T} V_{P N}+\Lambda_{N}^{2}\right) \mathcal{W}_{1 k, N} U_{f e}\right) k_{c}(t)+\psi(t)=0
\end{gathered}
$$

This is similar to (97), but with added weighting $\mathcal{F}_{\mathrm{ck}, N}$ and sub-system $\mathcal{W}_{1 k, N}$ present.

\subsection{The RS-GPC Control Signal}

The vector of future optimal control signals, to minimize the cost-index (70), follows from the condition for optimality (71). The vector of future optimal controls becomes: 


$$
k_{c}(t)=-\left(\Lambda_{K}^{2}+\Lambda_{D}^{2}+U_{f e}^{T} \mathcal{F}_{\mathrm{ck}, N} U_{f e}\right)^{-1}\left(\tilde{D}_{P t+k, N}^{0}+U_{f e}^{T}\left(V_{P N}^{T} V_{P N}+\Lambda_{N}^{2}\right) \mathcal{W}_{1 k, N} U_{f e} k_{c}(t)+\psi(t)\right)
$$

An alternative solution of equation (71), that may be easier for implementation, leads to the following gain vector expression:

$$
k_{c}(t)=-\left(\Lambda_{K}^{2}+\Lambda_{D}^{2}\right)^{-1}\left(\tilde{D}_{P t+k, N}^{0}+U_{f e}^{T}\left(\mathcal{F}_{c k, N}+\left(V_{P N}^{T} V_{P N}+\Lambda_{N}^{2}\right) \mathcal{W}_{1 k, N}\right) U_{f e} k_{c}(t)+\psi(t)\right)
$$

where $\tilde{D}_{P t+k, N}^{0}=P_{C N} D_{P t+k, N}+C_{\phi} \hat{x}(t+k \mid t)$.

\section{Theorem 3: Optimal RS-GPC Control Law}

Consider the plant, disturbance and output weighting models put in augmented state equation form (4) to (7), with input from the stable input plant dynamics $\mathcal{W}_{1 k}$. The costfunction to be minimized, with $N>0$, involving a sum of future cost-terms, is defined as follows:

$$
\begin{gathered}
J_{p}=E\left\{\Phi_{P t+k, N}^{0 T} \Phi_{P t+k, N}^{0} \mid t\right\} \\
\Phi_{P t+k, N}^{0}=P_{c N} E_{P t+k, N}+F_{c N}^{0} U_{t, N}^{0}+F_{c N}^{1} \tilde{k}_{c}(t)+F_{c v}^{2} \Delta \tilde{k}_{c}(t)+U_{f e}^{T} \mathcal{F}_{c k, N} U_{t, N}
\end{gathered}
$$

The error and control-input cost-function weightings are introduced as in the RS-GPC problem (34) and these determine the block matrix cost-index terms $P_{C N}=U_{f e}^{T} V_{P N}^{T}$ and $F_{c N}^{0}=U_{f e}^{T} \Lambda_{N}^{2}, \quad V_{P N}=C_{P N} B_{N}+E_{P N}$ and $C_{\phi}=P_{C N} C_{P N} A_{N}=U_{f e}^{T} V_{P N}^{T} C_{P N} A_{N}$. The gain weighting $F_{C N}^{1}=\Lambda_{K}^{2}$ and rate of change weighting $F_{C N}^{2}=\Lambda_{D}^{2}$ is also included, together with the dynamic control weighting function $\mathcal{F}_{\mathrm{ck}, N}$. The optimal gain vector, to minimize (74) becomes: 


$$
k_{c}(t)=-X_{F}^{-1}\left(P_{c N} D_{P t+k, N}+C_{\phi} \hat{x}(t+k \mid t)+U_{f e}^{T}\left(V_{P N}^{T} V_{P N}+\Lambda_{N}^{2}\right) \mathcal{W}_{1 k, N} U_{f e} k_{c}(t)+\psi(t)\right)
$$

where $\psi(t)=-\Lambda_{K}^{2} \bar{k}_{c}-\Lambda_{D}^{2} k_{c}(t-1)$ and $X_{F}=\Lambda_{K}^{2}+\Lambda_{D}^{2}+U_{f e}^{T} \mathcal{F}_{c k, N} U_{f e}$. The vector of future controls for prediction can be computed as $U_{t, N}=U_{f e}(t) k_{c}(t)$.

Solution: The proof follows by collecting results before the Theorem.

\section{Remarks on the solution:}

- The control law in Fig. 20, includes an internal model for the process, and is implemented using a receding horizon approach as in the above RS-GPC solution.

- The controller involves a Kalman predictor stage but the order of the Kalman filter only depends only on the delay free linear subsystems.

- Note $X_{N}$ from (43) the optimal gain (76) in the limiting case when the weighting $\mathcal{F}_{\mathrm{ck}, \mathrm{N}} \rightarrow 0$ and when $\mathcal{W}_{\mathrm{lk}, \mathrm{N}}=I$ becomes the same as for $R S-G P C$ control (88).

- Constraints may be applied at each sampling instant using quadratic programming to the magnitude of the controller gains, or their rate of change of gains. 


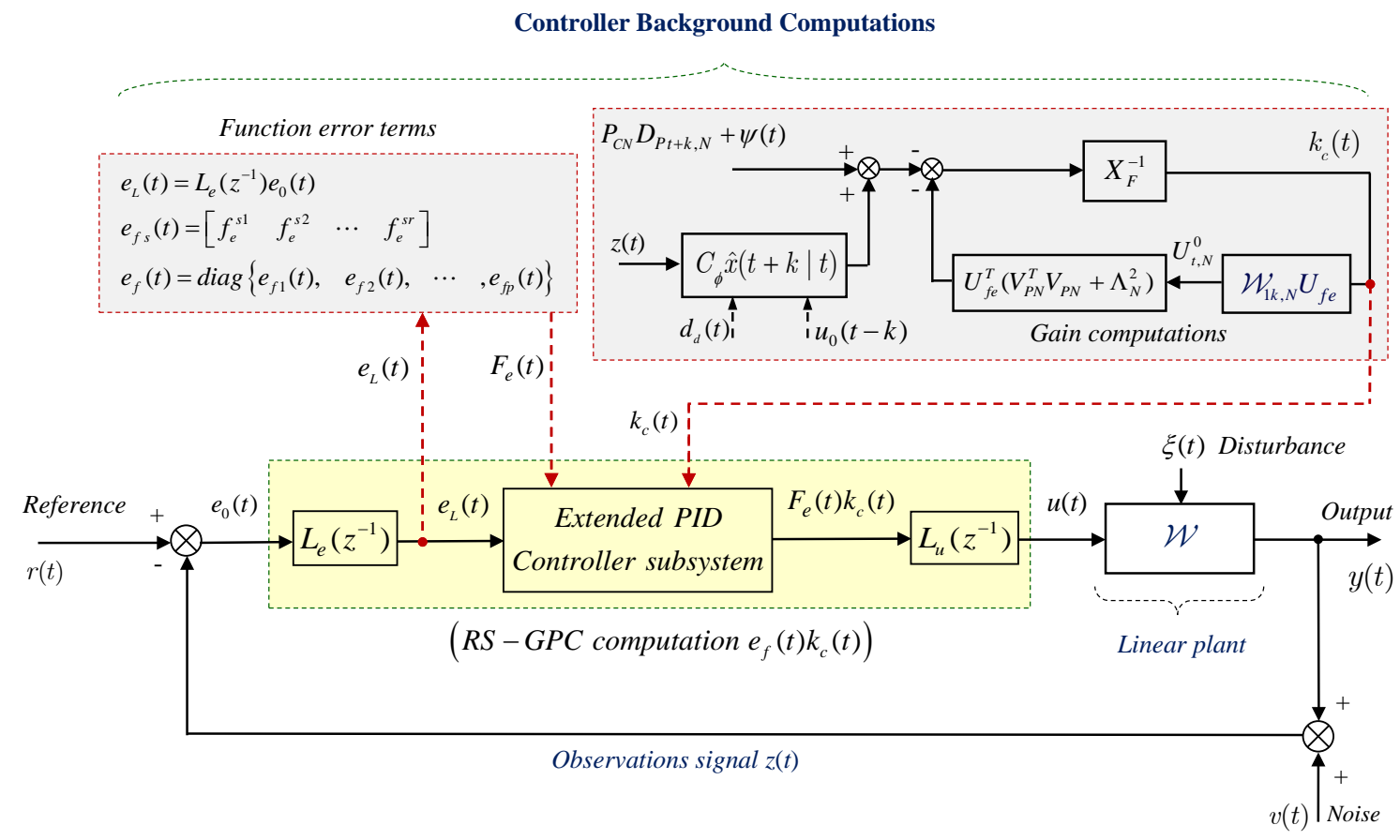

Figure 20. RS-GPC Feedback Control and Predictor for State and Unstructured Plant

\subsection{Stability of the Closed-Loop}

An expression may be derived for the control and output signals in closed-loop form, in terms of the exogenous inputs. Assume the stochastic external inputs are null and let $\Phi=\left(I-z^{-1} A\right)^{-1} z^{-1}$ then the state $x(t+1)=A x(t)+B u_{0}(t-k)+d_{d}(t)$ can be represented as follows:

$$
x(t)=\Phi\left(B u_{0}(t-k)+d_{d}(t)\right)
$$

The predicted-state, in this deterministic case $\hat{x}(t+k \mid t)=x(t+k)$. The cost-function can be simplified assuming $\Lambda_{K}^{2} \rightarrow 0$ and $\Lambda_{D}^{2} \rightarrow 0$. Recall from $U_{t, N}=U_{f e} k_{c}(t)$ where from (32):

$$
U_{f e}(t)=\left[\begin{array}{llll}
F_{e}^{T}(t) L_{u}^{T}\left(z^{-1}\right) & \cdots & \hat{F}_{e}^{T}(t+N-1) L_{u}^{T}\left(z^{-1}\right) & \hat{F}_{e}^{T}(t+N) L_{u}^{T}\left(z^{-1}\right)
\end{array}\right]^{T}
$$

Assume $N \geq N_{e}$ and $U_{f e}(t)$ and $U_{f e}^{T} \mathcal{F}_{\mathrm{c} k, N} U_{f e}$ are full-rank. For the absolute gain case $\bar{k}_{c}=0$ and $\psi(t)=-\Lambda_{K}^{2} \bar{k}_{c}-\Lambda_{D}^{2} k_{c}(t-1)=0$. Then from (71), the condition for optimality may be written as: 


$$
P_{C N} D_{P t+k, N}+P_{C N} C_{P N} A_{N} \hat{x}(t+k \mid t)+U_{f e}^{T}\left(\mathcal{F}_{c k, N}+\left(V_{P N}^{T} V_{P N}+\Lambda_{N}^{2}\right) \mathcal{W}_{1 k, N}\right) U_{f e} k_{c}(t)=0
$$

Recall (66) and $u_{0}(t)=C_{I 0} \mathcal{W}_{1 \mathrm{k}, \mathrm{N}} U_{f e}(t) k_{c}(t)$, where $C_{I 0}=[I, 0, \ldots, 0]$ and $C_{\phi}=U_{f e}^{T} V_{P N}^{T} C_{P N} A_{N}$ so that $\hat{x}(t+k \mid t)=\Phi\left(B u_{0}(t)+d_{d}(t+k)\right)$. The condition for optimality may be written as:

$$
\begin{gathered}
\left(U_{f e}^{T} \mathcal{F}_{\mathrm{ck}, N} U_{f e}+C_{\phi} \Phi B C_{I 0} \mathcal{W}_{1 \mathrm{k}, \mathrm{N}} U_{f e}+U_{f e}^{T}\left(V_{P N}^{T} V_{P N}+\Lambda_{N}^{2}\right) \mathcal{W}_{1 k, N} U_{f e}\right) k_{c}(t) \\
=-\left(P_{C N} D_{P t+k, N}+C_{\phi} \Phi d_{d}(t+k)\right)
\end{gathered}
$$

Recall from (30) the RS control $u(t)=L_{u} F_{e}(t) k_{c}(t)$. It follows that for the gain and control signals to be stable the operator in this equation, namely:

$$
\left(U_{f e}^{T} \mathcal{F}_{\mathrm{c} k, N} U_{f e}+C_{\phi} \Phi B C_{I 0} \mathcal{W}_{1 \mathrm{k}, \mathrm{N}} U_{f e}+U_{f e}^{T}\left(V_{P N}^{T} V_{P N}+\Lambda_{N}^{2}\right) \mathcal{W}_{1 k, N} U_{f e}\right)
$$

must have a stable inverse, which provides a necessary condition for stability.

\section{Concluding Remarks}

The example revealed some useful results and properties confirmed in other examples investigated. Firstly, the use of dynamic cost-function weightings was found to be important to provide flexibility and enable good transient response solutions to be obtained. Secondly on the question of implementation it was not obvious which of the two methods of implementing the controller (Absolute or Gain Deviation gains) was the most useful, since by careful tuning “good” results (not the same) could be achieved by either method, in for example step-response performance terms. It was concluded that the application would determine which was the most suitable.

Thirdly, it was found that the time-varying gains of the controller were found to remain constant much of the time, only changing with major disturbances or set-point changes. This is what was really needed and distinguished the work from much of the previous work on restricted structure control that assumed constant gains. The time-variation is what enables higher performance to be achieved. On the other hand, the robustness of 
low-order controllers relates to controllers with constant gains, and it is therefore useful that the gains do not normally vary widely for the types of system investigated. There was no problem experienced in implementing the constraints on gains that worked effectively but how useful this might be will depend upon hardware constraints in the application.

Fourthly, and the most important was the improved robustness achieved. Both for plant model mismatch and errors in transport-delay knowledge there was a considerable improvement over the classical control solution. Other examples have also indicated the approach may offer improved robustness, which was the main motivation for the work (it is recognized examples are by no means a proof). Finally, the example demonstrated that this type of model based predictive control solution that requires highly qualified design engineers can still be retuned using traditional tuning inputs like PID gain adjustments.

By combining the two most successful control techniques used in industry a design method has been produced with good potential in applications. The work is novel since the previous work on $R S$-controllers has not included the nonlinear subsystem, or the potential to use either soft or hard constraints on the controller gains.

The advantages of this RS-GPC approach may be summarized as follows:

- The computations will normally be less than traditional MPC since the main matrix to be inverted depends on the number of gains used in the RS-controller, which are often less than the control horizon needed for good performance.

- The PID controller is very effective and seems to have inherited the natural robustness of low-order controllers. Moreover, adding terms to its dynamic order should give it greater flexibility. 
- Parameterising the controller in terms of a set of linear dynamic functions (like PID function terms), multiplied by unknown gains is simple and computation of the gains by predictive control is straightforward.

- Using prediction enables information on future reference and disturbance changes to be included. The controller structure can include natural feed-forward terms (from known disturbances), and it provides transport-delay compensation.

- A higher performance than PID should be possible, since time-varying gains and more functions provide added refinement and flexibility. This should be particularly beneficial for multivariable applications (Majecki et.al. 2015, 2017).

- Provides a way of auto-tuning or benchmarking low-order designs like a PID controller, assuming a model is available.

- If the optimal gains are computed and simply stored for operation in different regions it provides a simple solution for implementation using scheduling.

\section{References}

Clarke, D.W., C. Mohtadi and P.S. Tuffs, (1987), Generalized predictive control - Part 1, The basic algorithm, Part 2, Extensions and interpretations, Automatica, 23, 2, pp.137148.

Clarke, D.W., and C. Montadi, (1989), Properties of generalised predictive control, Automatica, Vol. 25, No. 6, pp. 859-875.

Diaz-Rodriguez, I. D., and S. P. Bhattacharyya, (2016), PI Controller Design in the Achievable Gain-Phase Margin Plane, IEEE 55th Conference on Decision and Control, December 12-14, 2016, Las Vegas. 
Eielsen, A. A.,Vagia, M., Gravdahl, J. T., Pettersen, K. Y., (2013), Fixed-Structure, LowOrder Damping and Tracking Control Schemes for Nano positioning, Proceedings of the 6th IFAC Symposium on Mechatronic Systems, Zhejiang University, Hangzhou, China. Grimble, M.J. and Johnson, M. A., (1988), Optimal control and stochastic estimation theory, Parts I and II. Wiley.

Grimble, M.J., (2000), Restricted structure LQG optimal control for continuous-time systems, IEE Proc., Control Theory and Applications, Vol. 147, No. 2.

Grimble, M. J., (2004a), Optimal restricted structure control with pre-specified gain or phase margins, IEE Proceedings, Control Theory and Applications, Vol. 151, no. 3, pp. 271-277.

Grimble, M. J., (2004b), Robustness of full-order and restricted-structure optimal control systems, Int. Journal of Systems Science, vol. 35, no. 6, pp. 375-388.

Grimble, M. J., (2004c), Restricted structure predictive optimal control, Optimal Control Applications and Methods, vol. 25, no. 3, pp. 107-145.

Grimble, M J, (2005), Non-linear generalised minimum variance feedback, feedforward and tracking control, Automatica, Vol. 41, pp. 957-969.

Grimble, M.J., and Majecki, P., (2010a), State-Space Approach to Nonlinear Predictive Generalized Minimum Variance Control, International Journal of Control, Volume 83 Issue 8, pp. 1529-1547.

Grimble, M.J., and Majecki, P., (2010b), Polynomial Approach to Nonlinear Predictive Generalized Minimum Variance Control, IET Control Theory and Appl., Vol.4, Issue No. 3, pp. $411-424$.

Guo W., W. Wang, and X. Qiu, (2008), An improved generalised predictive control algorithm based on PID, Int. Conf. on Intelligent Computation Techn. and Automation, IEEE Computer Society, pp. 299-303. 
Katebi M.R. and M.H. Moradi, (2001), Predictive controllers, IEEE Proceedings on Control Theory and Applications Vol.148, No 6, November, pp. 478-487.

Khadir M, T, and J. V. Ringwood, (2008), Extension of First Order Predictive Functional Controllers to Handle Higher Order Internal Models, Int. J. Appl. Math. Computing Sci., Vol. 18, No. 2, pp. 229-239.

Kwon W.H. and Pearson, A.E., (1977), A modified quadratic cost problem and feedback stabilization of a linear system, IEEE Trans. on Automatic Control, Vol. AC-22, No. 5, pp. 838-842.

Majecki, P, van der Molen, G.M., Grimble, M J., Haskara I, Hu Y, Chen-Fang Chang, (2015), Real-Time Predictive Control for SI Engines Using Linear Parameter-Varying Models, 5th IFAC Conference on Nonlinear Model Predictive Control, Seville, 2015 and IFAC-Papers On-Line, Volume 48, Issue 23, 2015, pp. 94-101.

Majecki, P., Grimble, M. J., Haskara, I., Hu, Y., Chang, C. F., (2017), Total Engine Optimization and Control for SI Engines Using Linear Parameter-Varying Models, American Control Conference, Seattle, WA, USA, May 24-26.

Millar R.M., K.E. Kwok, S.L. Shah and R. K. Wood, (1996), Development of a Stochastic Predictive PID Controller, Proc. American Control Conference, Seattle, Washington, June, pp. 4204-4208.

Moradi, M. H, Katebi M R and M. A. Johnson, (2001), Predictive PID Control: A new algorithm, IECON' 01: The 27th Annual Conference of the IEEE Industrial Electronics Society, pp. 764-769.

Ordys, A.W., and D.W. Clarke, (1993), A state-space description for GPC controllers, Int. J. Systems Science, Vol. 23, No. 2.

Richalet J., A. Rault, J. L. Testud, J. Papon (1978), Model predictive heuristic control applications to industrial processes, Automatica, 14, pp. 413-428. 
Richalet, J., (1993), Industrial applications of model based predictive control, Automatica, Vol. 29, No. 8, pp. 1251-1274.

Richalet, J., (1998), La commande predictive, Techniques de l'Ingenieur Traite Mesure et Control, pp. 1-17.

Rossiter, J.A., and Richalet, J., (2002), Handling constraints with predictive functional control of unstable processes, American Control Conference, Anchorage, pp. 4746-4751. Sato T, (2010), Design of a GPC-based PID controller for controlling a weigh feeder, Control Engineering Practice, 18, pp. 105-1103.

Uduehi, D, A. Ordys and M.J. Grimble, (2002), Multivariable PID controller design using online generalised predictive control optimisation, Proc. IEEE International Conference on Control Applications, September 18-20, 2002, Glasgow, pp. 272-277.

\section{Appendix 1: Notation and Signal Dimensions}

The signals and dimensions may be listed as follows:

$x(t)$ : Vector of $n$ system states in the linear plant subsystem and the disturbance model.

$u_{0}(t)$ : Vector of $m_{0}$ input signals to the linear output subsystem.

$u(t)$ : Vector of $m$ controls applied to the input subsystem, representing the plant input.

$y_{m}(t)$ : Vector of $r$ plant output signals that are measured.

$z_{m}(t)$ : Vector of $r$ observations or measured plant outputs including measurement noise.

$r_{w}(t)$ : Vector of $r$ plant set-point or reference signals (known $k+N$ steps ahead).

$r(t)$ : Vector of $r$ plant set-point or deterministic reference model output signals.

$e_{0}(t)$ : Vector of $r$ noisy error (reference-observations) signals.

$e_{p}(t)$ : Vector of $m$ inferred output or error signals to be controlled including weightings.

$d_{m}(t)$ : Vector of $r$ known output disturbance signal values. 
$d_{p}(t)$ : Vector of $r$ known inferred output disturbance signal values.

$d_{d}(t)$ : Vector of $q$ known input disturbance signal values.

\section{Appendix 2: Block Matrix Definitions}

The following vectors and block-matrices may be defined for the general case $N>0$ as:

$$
\begin{gathered}
C_{P N}=\operatorname{diag}\left\{C_{p}, C_{p}, \ldots, C_{p}\right\} \quad \text { and } \quad E_{P N}=\operatorname{diag}\left\{E_{p}, E_{p}, \ldots, E_{p}\right\} \quad(N+1 \text { square }) \\
A_{N}=\left[\begin{array}{c}
I \\
A \\
A^{2} \\
\vdots \\
A^{N}
\end{array}\right], \quad B_{N}=\left[\begin{array}{ccccc}
0 & 0 & \cdots & 0 & 0 \\
B & 0 & \cdots & 0 \\
\vdots & B & \ddots & \vdots \\
A^{N-1} B & A^{N-2} B & \cdots & B & 0
\end{array}\right], D_{N}=\left[\begin{array}{cccc}
0 & 0 & \cdots & 0 \\
D & 0 & \ddots & 0 \\
A D & D & & \\
\vdots & & \ddots & 0 \\
A^{N-1} D & A^{N-2} D & \cdots & D
\end{array}\right] \\
D_{P t, N}=\left[\begin{array}{c}
d_{p d}(t) \\
d_{p d}(t+1) \\
d_{p d}(t+2) \\
\vdots \\
d_{p d}(t+N)
\end{array}\right], \quad W_{t, N}=\left[\begin{array}{c}
\xi(t) \\
\xi(t+1) \\
\vdots \\
\xi(t+N-1)
\end{array}\right], \quad U_{t, N}^{0}=\left[\begin{array}{c}
u_{0}(t) \\
u_{0}(t+1) \\
\vdots \\
u_{0}(t+N)
\end{array}\right]
\end{gathered}
$$

For the special case of a single-step cost-function $N=0$ define $A_{N}=I, \quad B_{N}=D_{N}=0$, $C_{P N}=C_{p}, E_{P N}=E_{p}$. The $W_{t, N}$ denotes a vector of white noise inputs and $U_{t, N}^{0}$ denotes a block vector of future input signals.

\section{Appendix 3: Parameterizing the RS-GPC Controller}

The function $f_{j}\left(z^{-1}, k_{j}(t)\right)$ and gains $k_{j}(t)$ are defined to have the following matrix forms:

$$
f_{j}\left(z^{-1}, k_{j}(t)\right)=\left[\begin{array}{cccc}
f_{11}^{j}\left(z^{-1}\right) k_{11}^{j}(t) & \ldots & & f_{1 r}^{j}\left(z^{-1}\right) k_{1 r}^{j}(t) \\
\vdots & f_{22}^{j}\left(z^{-1}\right) k_{22}^{j}(t) & & \vdots \\
& & \ddots & \\
f_{m 1}^{j}\left(z^{-1}\right) k_{m 1}^{j}(t) & \cdots & & f_{m r}^{j}\left(z^{-1}\right) k_{m r}^{j}(t)
\end{array}\right]
$$




$$
k_{j}=\left[\begin{array}{cccc}
k_{11}^{j} & k_{12}^{j} & \cdots & k_{1 r}^{j} \\
k_{21}^{j} & k_{22}^{j} & & \vdots \\
\vdots & & \ddots & \\
k_{m 1}^{j} & \cdots & & k_{m r}^{j}
\end{array}\right]
$$

In the special case when these matrices can be defined to be diagonal:

$$
f_{j}\left(z^{-1}, k_{j}(t)\right)=\operatorname{diag}\left\{f_{11}^{j}\left(z^{-1}\right) k_{11}^{j}(t), \quad f_{22}^{j}\left(z^{-1}\right) k_{22}^{j}(t), \quad \cdots \quad, f_{m m}^{j}\left(z^{-1}\right) k_{m m}^{j}(t)\right\}
$$

where $k_{j}=\operatorname{diag}\left\{k_{11}^{j}, \quad k_{22}^{j}, \cdots, k_{m m}^{j}\right\}$. The proposed controller structure involves the sum of vector functions that form the control signal. These might be the sum of proportional, integral and filtered derivative terms for each of the channels. From (26):

$$
u(t)=L_{u}\left(z^{-1}\right)\left(f_{1}\left(z^{-1}, k_{1}(t)\right) e_{L}(t)+f_{2}\left(z^{-1}, k_{2}(t)\right) e_{L}(t)+\cdots+f_{N_{e}}\left(z^{-1}, k_{N_{e}}(t)\right) e_{L}(t)\right)
$$

The functions $f_{j}\left(z^{-1}, k_{j}(t)\right)$ and the gains $k_{j}(t)$ determine the controller structure and the controller gains, respectively. The gains $k_{j}(t)$ represent a set of time-varying gain vectors for the multivariable controller, with a total of $N_{e}$ function block terms.

Let the vector of weighted errors $e_{L}(t)=L_{e}\left(z^{-1}\right) e_{0}(t)$ may be written in terms of the scalar signals for each error channel as:

$$
e_{L}^{T}(t)=\left[\begin{array}{llll}
e_{1}^{L}(t) & e_{2}^{L}(t) & \cdots & e_{r}^{L}(t)
\end{array}\right]
$$

Each of the terms in the summation in (26) has the form $f_{j}\left(z^{-1}, k_{j}(t)\right) e_{L}(t)$. From (77) the contribution of the $j$ th function term in each channel, can be written as:

$$
f_{j}\left(z^{-1}, k_{j}(t)\right) e_{L}(t)=\left[\begin{array}{cccc}
f_{11}^{j}\left(z^{-1}\right) k_{11}^{j}(t) & \ldots & & f_{1 r}^{j}\left(z^{-1}\right) k_{1 r}^{j}(t) \\
\vdots & f_{22}^{j}\left(z^{-1}\right) k_{22}^{j}(t) & & \vdots \\
& & \ddots & \\
f_{m 1}^{j}\left(z^{-1}\right) k_{m 1}^{j}(t) & \ldots & & f_{m r}^{j}\left(z^{-1}\right) k_{m r}^{j}(t)
\end{array}\right]\left[\begin{array}{c}
e_{1}^{L}(t) \\
e_{2}^{L}(t) \\
\vdots \\
e_{r}^{L}(t)
\end{array}\right]
$$


The expression (26) for the control signal may now be written (from (78)) as:

$$
u(t)=L_{u}\left(z^{-1}\right)\left[\begin{array}{c}
\sum_{j=1}^{N_{e}}\left\{f_{11}^{j}\left(z^{-1}\right) k_{11}^{j} e_{1}^{L}(t)+f_{12}^{j}\left(z^{-1}\right) k_{12}^{j} e_{2}^{L}(t)+\ldots+f_{1 r}^{j}\left(z^{-1}\right) k_{1 r}^{j} e_{r}^{L}(t)\right\} \\
\sum_{j=1}^{N_{e}}\left\{f_{21}^{j}\left(z^{-1}\right) k_{21}^{j} e_{1}^{L}(t)+f_{22}^{j}\left(z^{-1}\right) k_{22}^{j} e_{2}^{L}(t)+\ldots+f_{2 r}^{j}\left(z^{-1}\right) k_{2 r}^{j} e_{r}^{L}(t)\right\} \\
\vdots \\
\vdots \\
\sum_{j=1}^{N_{e}}\left\{f_{m 1}^{j}\left(z^{-1}\right) k_{m 1}^{j} e_{1}^{L}(t)+f_{m 2}^{j}\left(z^{-1}\right) k_{m 2}^{j} e_{2}^{L}(t)+\ldots+f_{m r}^{j}\left(z^{-1}\right) k_{m r}^{j} e_{r}^{L}(t)\right\}
\end{array}\right]
$$

\section{Control signal for parameterized controller}

The expression (79) provides a valid parameterization of the controller but it needs to be in a more convenient form for the optimization of the gains. An expression is required where the gains are collected in a vector to be optimized. Motivated by the summation terms in (79) define:

$$
f_{e}^{i s}=\left[f_{i s}^{1}\left(z^{-1}\right) e_{s}^{L}(t) \quad f_{i s}^{2}\left(z^{-1}\right) e_{s}^{L}(t) \ldots . \quad f_{i s}^{N_{e}}\left(z^{-1}\right) e_{s}^{L}(t)\right]
$$

and the gain vector:

$$
k_{c}^{i s}=\left[\begin{array}{llll}
k_{i s}^{1 T} & k_{i s}^{2 T} & \cdots & k_{i s}^{N_{e} T}
\end{array}\right]^{T}
$$

Thus, from (80) and (81) obtain:

$$
f_{e}^{i s} k_{c}^{i s}=\left[\begin{array}{llll}
f_{i s}^{1}\left(z^{-1}\right) e_{s}^{L}(t) & f_{i s}^{2}\left(z^{-1}\right) e_{s}^{L}(t) \ldots . . & f_{i s}^{N_{e}}\left(z^{-1}\right) e_{s}^{L}(t)
\end{array}\right]\left[\begin{array}{c}
k_{i s}^{1} \\
k_{i s}^{2} \\
\vdots \\
k_{i s}^{N_{e}}
\end{array}\right]=\sum_{j=1}^{N_{e}}\left\{f_{i s}^{j}\left(z^{-1}\right) e_{s}^{L}(t) k_{i s}^{j}\right\}
$$

That is, the contribution to the control in channel $i$, corresponding to the error in output channel s, can be obtained as:

$$
f_{e}^{i s} k_{c}^{i s}=\sum_{j=1}^{N_{e}}\left\{f_{i s}^{j}\left(z^{-1}\right) e_{s}^{L}(t) k_{i s}^{j}\right\}
$$


The parameterized control in the general case, follows from (79) and (82):

$$
u(t)=L_{u}\left(z^{-1}\right)\left[\begin{array}{c}
f_{e}^{11} k_{c}^{11}+f_{e}^{12} k_{c}^{12}+\ldots+f_{e}^{1 r} k_{c}^{1 r} \\
f_{e}^{21} k_{c}^{21}+f_{e}^{22} k_{c}^{22}+\ldots+f_{e}^{2 r} k_{c}^{2 r} \\
\vdots \\
f_{e}^{m 1} k_{c}^{m 1}+f_{e}^{m 2} k_{c}^{m 2}+\ldots+f_{e}^{m r} k_{c}^{m r}
\end{array}\right]
$$

The scalar gains may now be collected in one total vector as:

$$
k_{c}(t)=\left[\begin{array}{llll}
k_{c 1}^{T} & k_{c 2}^{T} & \cdots & k_{c m}^{T}
\end{array}\right]^{T}, \quad \text { where } \quad k_{c i}(t)=\left[\begin{array}{llll}
k_{c}^{i 1} & k_{c}^{i 2} & \cdots & k_{c}^{i r}
\end{array}\right]^{T}
$$

The total gain-vector has $r \times m \times N_{e}$ rows which may be written as:

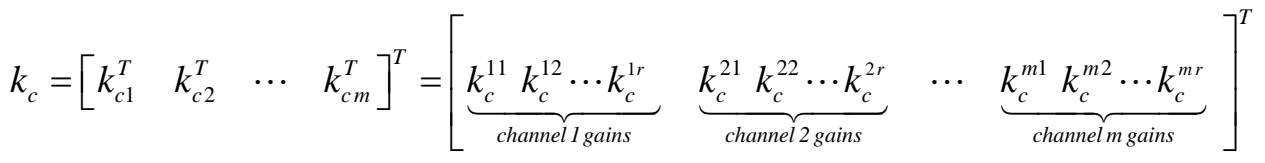

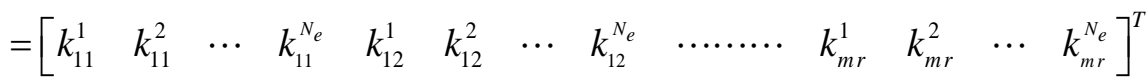

The gain-vector $k_{c}$ includes the functional controller gains for each channel listed in order. The restricted structure controller may now be represented as $u(t)=L_{u} F_{e}(t) k_{c}(t)$.

\section{Appendix 4: Square of Sum Optimization Problem}

The following proof for Theorem 2 shows that an equivalent minimum variance problem may be solved which has the same solution as the RS-GPC design, which is needed for motivating the nonlinear control problem solved in the last section. Let the constant positive-definite, real symmetric matrix in (43) be factorized as:

$$
Y^{T} Y=X_{N}=U_{f e}^{T}\left(V_{P N}^{T} V_{P N}+\Lambda_{N}^{2}\right) U_{f e}+\Lambda_{K}^{2}+\Lambda_{D}^{2}
$$

Note from (33), $U_{t, N}=U_{f e}(t) k_{c}(t)$ and (50) $\tilde{D}_{P t+k, N}^{0}=P_{C N} \tilde{D}_{P t+k, N}$. From (47) the criterion: 


$$
\begin{gathered}
J=\tilde{D}_{P t+k, N}^{T} \tilde{D}_{P t+k, N}+k_{c}^{T}(t) P_{c N} \tilde{D}_{P t+k, N}+\tilde{D}_{P t+k, N}^{T} P_{c N}^{T} k_{c}(t)+\psi(t)^{T} k_{c}(t)+k_{c}^{T}(t) \psi(t)+k_{c}^{T}(t) X_{N} k_{c}(t)+\bar{J}_{0} \\
=\tilde{D}_{P t+k, N}^{T} \tilde{D}_{P t+k, N}+k_{c}^{T}(t)\left(P_{C N} \tilde{D}_{P t+k, N}+\psi(t)\right) \\
+\left(\tilde{D}_{P t+k, N}^{T} P_{C N}^{T}+\psi(t)^{T}\right) k_{c}(t)+k_{c}^{T}(t) Y^{T} Y k_{c}(t)+\bar{J}_{0}
\end{gathered}
$$

Recall $\tilde{D}_{P t+k, N}^{0}=P_{C N} \tilde{D}_{P t+k, N}$ and then by completing the squares:

$$
J=\left(\left(\tilde{D}_{P t+k, N}^{0 T}+\psi(t)^{T}\right) Y^{-1}+k_{c}^{T}(t) Y^{T}\right)\left(Y^{-T}\left(\tilde{D}_{P t+k, N}^{0}+\psi(t)\right)+Y k_{c}(t)\right)+J_{\text {min }}
$$

where the minimum-cost, including terms that do not involve $k_{c}(t)$, follows as:

$$
J_{\min }=\tilde{D}_{P t+k, N}^{T} \tilde{D}_{P t+k, N}-\left(\tilde{D}_{P t+k, N}^{0 T}+\psi(t)^{T}\right) Y^{-1} Y^{-T}\left(\tilde{D}_{P t+k, N}^{0}+\psi(t)\right)+\bar{J}_{0}
$$

From inspection of the cost $J$ it is also useful to define the signal:

$$
\hat{\Phi}_{P t+k, N}=Y^{-T}\left(\tilde{D}_{P t+k, N}^{0}+\psi(t)\right)+Y k_{c}(t)
$$

The cost-function (54) may therefore be written in an equivalent form as:

$$
J=\hat{\Phi}_{P t+k, N}^{T} \hat{\Phi}_{P t+k, N}+J_{\text {min }}(t)
$$

Observe that the last term $J_{\min }(t)$ in equation does not depend upon current control action and the optimal control is found by setting the squared term in (86) to zero. That is, the optimal control is obtained by setting the term containing the predicted inferred output $\hat{\Phi}_{P t+k, N}$ to zero. Also note $P_{c N}=U_{f e}^{T} V_{P N}^{T}$ and the minimum cost $J_{\text {min }}(t)$ therefore depends on $U_{f e}(t)$, which is a function of the controller parameterization.

Writing $X_{N}=Y^{T} Y$ the condition for optimality in this problem can be expressed as: 


$$
\tilde{D}_{P t+k, N}^{0}+\psi(t)+X_{N} k_{c}(t)=0
$$

and the optimal gain vector:

$$
\begin{gathered}
k_{c}(t)=-\left(Y^{T} Y\right)^{-1}\left(\tilde{D}_{P t+k, N}^{0}+\psi(t)\right) \\
=-X_{N}^{-1}\left(P_{c N} D_{P t+k, N}+C_{\phi} \hat{x}(t+k \mid t)+\psi(t)\right)
\end{gathered}
$$

This solution (88) is the same control as in (51). That is, the RS-GPC optimal controller for the above system and cost (54), is the same as the controller to minimise the Euclidean norm of the signal $\hat{\Phi}_{t+k, N}$, defined in (86).

\section{Modified Cost-Function Generating RS-GPC Control}

This result motivates the definition of a different multi-step minimum-variance cost problem that has the same solution (still considering case $\mathcal{W}_{1 \mathrm{k}}=\mathrm{I}$ ). Consider a new signal to be minimised involving a weighted sum of error and inputs as in NGMV control of the form $\phi(t+k)=P_{c} e(t+k)+F_{\mathrm{c} 0} u_{0}(t)$. The vector of future values of this signal, for a multi-step cost-index, may be defined as:

$$
\Phi_{P t+k, N}=P_{C N} E_{P t+k, N}+F_{C N}^{0} U_{t, N}^{0}+F_{C N}^{1} \tilde{k}_{c}(t)+F_{C N}^{2} \Delta \tilde{k}_{c}(t)
$$

Introduce cost-weightings, motivated by the RS-GPC weightings, to have the form:

$$
P_{c N}=U_{f e}^{T}(t) V_{P N}^{T}, \quad F_{c N}^{0}=U_{f e}^{T}(t) \Lambda_{N}^{2} \quad \text { and } \quad F_{c N}^{1}=\Lambda_{K}^{2}, \quad F_{c N}^{2}=\Lambda_{D}^{2}
$$

where $\Delta \tilde{k}_{c}(t)=\tilde{k}_{c}(t)-\tilde{k}_{c}(t-1)$. This choice is justified by the results in Theorem 2 that follows below. The new multi-step cost-function, using the vector of signals (89):

$$
\tilde{J}=E\left\{\tilde{J}_{t}\right\}=E\left\{\Phi_{P t+k, N}^{T} \Phi_{P t+k, N} \mid t\right\}
$$




\section{Solution}

The solution to this problem for the minimization of the variance of $\Phi_{P t+k, N}$ may be considered, noting (89) and substituting for $E_{P t+k, N}=\hat{E}_{P t+k, N}+\tilde{E}_{P t+k, N}$. From (89),

$$
\begin{aligned}
& \Phi_{P t+k, N}=P_{c N} E_{P t+k, N}+F_{c N}^{0} U_{t, N}^{0}+F_{c N}^{1} \tilde{k}_{c}+F_{c N}^{2} \Delta \tilde{k}_{c} \\
= & P_{C N} \hat{E}_{P t+k, N}+F_{C N}^{0} U_{t, N}^{0}+F_{C N}^{1} \tilde{k}_{c}+F_{C N}^{2} \Delta \tilde{k}_{c}+P_{C N} \tilde{E}_{P t+k, N}
\end{aligned}
$$

This expression may be written in terms of the estimate and the estimation error as:

$$
\Phi_{P t+k, N}=\hat{\Phi}_{P t+k, N}+\tilde{\Phi}_{P t+k, N}
$$

Clearly the predicted signal and the prediction error:

$$
\begin{gathered}
\hat{\Phi}_{P t+k, N}=P_{C N} \hat{E}_{P t+k, N}+F_{C N}^{0} U_{t, N}^{0}+F_{C N}^{1} \tilde{k}_{c}+F_{C N}^{2} \Delta \tilde{k}_{c} \\
\tilde{\Phi}_{P t+k, N}=P_{C N} \tilde{E}_{P t+k, N}
\end{gathered}
$$

The performance index (91) may therefore be expanded and written as:

$$
\tilde{J}=E\left\{\tilde{J}_{t}\right\}=E\left\{\Phi_{P t+k, N}^{T} \Phi_{P t+k, N} \mid t\right\}=E\left\{\left(\hat{\Phi}_{P t+k, N}+\tilde{\Phi}_{P t+k, N}\right)^{T}\left(\hat{\Phi}_{P t+k, N}+\tilde{\Phi}_{P t+k, N}\right) \mid t\right\}
$$

The terms in the performance index (91) can be simplified, recalling the optimal estimate $\hat{E}_{P t+k, N}$ and the estimation error $\tilde{E}_{P t+k, N}$ are orthogonal. Thus, obtain:

$$
\begin{gathered}
\tilde{J}=E\left\{\hat{\Phi}_{P t+k, N}^{T} \hat{\Phi}_{P t+k, N} \mid t\right\}+E\left\{\hat{\Phi}_{P t+k, N}^{T} \tilde{\Phi}_{P t+k, N} \mid t\right\}+E\left\{\tilde{\Phi}_{P t+k, N}^{T} \hat{\Phi}_{P t+k, N} \mid t\right\}+E\left\{\tilde{\Phi}_{P t+k, N}^{T} \tilde{\Phi}_{P t+k, N} \mid t\right\} \\
=\hat{\Phi}_{P t+k, N}^{T} \hat{\Phi}_{P t+k, N}+\tilde{J}_{1}(t)
\end{gathered}
$$

The last cost-term (95), that is independent of control action, may be written as: 


$$
\tilde{J}_{1}(t)=E\left\{\tilde{\Phi}_{P t+k, N}^{T} \tilde{\Phi}_{P t+k, N} \mid t\right\}=E\left\{\tilde{E}_{P t+k, N}^{T} P_{C N}^{T} P_{C N} \tilde{E}_{P t+k, N} \mid t\right\}
$$

The signals $\hat{\Phi}_{P t+k, N}$ may be simplified, by substituting for $\hat{E}_{P t+k, N}$ from (21). Thence,

$$
\begin{gathered}
\hat{\Phi}_{P t+k, N}=P_{C N} \hat{E}_{P t+k, N}+F_{c N}^{0} U_{t, N}^{0}+F_{c N}^{1} \tilde{k}_{c}+F_{c N}^{2} \Delta \tilde{k}_{c} \\
=P_{c N}\left(D_{P t+k, N}+C_{P N} A_{N} \hat{x}(t+k \mid t)+V_{P N} U_{t, N}^{0}\right)+F_{c N}^{0} U_{t, N}^{0}+F_{c N}^{1} \tilde{k}_{c}+F_{c N}^{2} \Delta \tilde{k}_{c} \\
=\tilde{D}_{P t+k, N}^{0}+P_{c N} V_{P N} U_{t, N}^{0}+F_{c N}^{0} U_{t, N}^{0}+F_{c N}^{1} \tilde{k}_{c}+F_{c N}^{2} \Delta \tilde{k}_{c}
\end{gathered}
$$

Substituting from (33) the $U_{t, N}=U_{f e}(t) k_{c}(t)=U_{f e}(t)\left(\bar{k}_{c}+\tilde{k}_{c}(t)\right)$ and recalling the definitions of weights $F_{c N}^{0}=U_{f e}^{T} \Lambda_{N}^{2}, F_{C N}^{1}=\Lambda_{K}^{2}$ and $F_{c N}^{2}=\Lambda_{D}^{2}$. Also noting (52) obtain:

$$
\begin{gathered}
\hat{\Phi}_{P t+k, N}=\tilde{D}_{P t+k, N}^{0}+P_{C N} V_{P N} U_{t, N}^{0}+U_{f e}^{T} \Lambda_{N}^{2} U_{f e} k_{c}+\Lambda_{K}^{2} \tilde{k}_{c}+\Lambda_{D}^{2} \Delta \tilde{k}_{c} \\
=\tilde{D}_{P t+k, N}^{0}+\left(U_{f e}^{T} V_{P N}^{T} V_{P N} U_{f e}+U_{f e}^{T} \Lambda_{N}^{2} U_{f e}\right) k_{c}(t)+\Lambda_{K}^{2} \tilde{k}_{c}+\Lambda_{D}^{2}\left(\tilde{k}_{c}(t)-\tilde{k}_{c}(t-1)\right)
\end{gathered}
$$

Recall from (37) $\Delta \tilde{k}_{c}(t)=k_{c}(t)-k_{c}(t-1)$ and from (29) $\tilde{k}_{c}(t)=k_{c}(t)-\bar{k}_{c}$ then,

$$
\begin{gathered}
\hat{\Phi}_{P t+k, N}=\tilde{D}_{P t+k, N}^{0}+\left(U_{f e}^{T}\left(V_{P N}^{T} V_{P N}+\Lambda_{N}^{2}\right) U_{f e}+\Lambda_{K}^{2}+\Lambda_{D}^{2}\right) k_{c}(t)-\Lambda_{K}^{2} \bar{k}_{c}-\Lambda_{D}^{2} k_{c}(t-1) \\
=\tilde{D}_{P t+k, N}^{0}+X_{N} k_{c}(t)+\psi(t)
\end{gathered}
$$

The optimal control must set the first squared term in (95) to zero $\hat{\Phi}_{P t+k, N}=0$. The optimal gains therefore follow by setting (97) to zero, giving:

$$
k_{c}(t)=-X_{N}^{-1}\left(\tilde{D}_{P t+k, N}^{0}+\psi(t)\right)=-X_{N}^{-1}\left(P_{C N} D_{P t+k, N}+C_{\phi} \hat{x}(t+k \mid t)+\psi(t)\right)
$$

This expression is the same as would be obtained from the condition for optimality (87), 
derived for the equivalent optimization problem. The solution (98) is the same as the vector of RS-GPC control gains, as summarised in Theorem 2 which follows. 